CLNS 06/1971

FERMILAB-PUB-06-242-T

SFB/CPP-06-32

SI-HEP-2006-09

July 20, 2006

\title{
Factorization and Momentum-Space Resummation in Deep-Inelastic Scattering
}

\author{
Thomas Becher ${ }^{a}$, Matthias Neubert $^{b, c}$, And Ben D. PecjaK ${ }^{d}$ \\ ${ }^{a}$ Fermi National Accelerator Laboratory \\ P.O. Box 500, Batavia, IL 60510, U.S.A. \\ ${ }^{b}$ Institute for High-Energy Phenomenology \\ Newman Laboratory for Elementary-Particle Physics, Cornell University \\ Ithaca, NY 14853, U.S.A. \\ ${ }^{c}$ Institut für Physik (ThEP), Johannes Gutenberg-Universität \\ D-55099 Mainz, Germany \\ ${ }^{d}$ Theoretische Physik 1, Fachbereich Physik, Universität Siegen \\ D-57068 Siegen, Germany
}

\begin{abstract}
Renormalization-group methods in soft-collinear effective theory are used to perform the resummation of large perturbative logarithms for deep-inelastic scattering in the threshold region $x \rightarrow 1$. The factorization theorem for the structure function $F_{2}\left(x, Q^{2}\right)$ for $x \rightarrow 1$ is rederived in the effective theory, whereby contributions from the hard scale $Q^{2}$ and the jet scale $Q^{2}(1-x)$ are encoded in Wilson coefficients of effective-theory operators. Resummation is achieved by solving the evolution equations for these operators. Simple analytic results for the resummed expressions are obtained directly in momentum space, and are free of the Landau-pole singularities inherent to the traditional momentspace results. We show analytically that the two methods are nonetheless equivalent order by order in the perturbative expansion, and perform a numerical comparison up to next-to-next-to-leading order in renormalization-group improved perturbation theory.
\end{abstract}




\section{Introduction}

It is well known that fixed-order perturbation theory is not reliable for quantities involving several disparate scales. In such cases, higher-order corrections are enhanced by large logarithms of scale ratios. The standard solution to this problem is to split the calculation into a series of single-scale problems by successively integrating out the physics associated with the largest remaining scale. Perturbative logarithms are then resummed by renormalization-group (RG) evolution from the larger scales to the smaller ones. For collider processes, resummation is traditionally performed by other means, since it was not always clear how to systematically integrate out the physics associated with high scales in such cases.

The simplest example of a high-energy process with a scale hierarchy which necessitates resummation is deep-inelastic scattering (DIS) in the threshold region. As the Bjorken scaling variable $x \rightarrow 1$, the invariant mass of the hadronic system produced in the decay, $M_{X}=$

$Q \sqrt{\frac{1-x}{x}}$ (neglecting the nucleon mass), becomes much smaller than the momentum transfer Q. The presence of the two scales is manifest in the QCD factorization theorem [1, 2, 3]

$$
F_{2}^{\mathrm{ns}}\left(x, Q^{2}\right)=H\left(Q^{2}, \mu\right) Q^{2} \int_{x}^{1} \frac{d z}{z} J\left(Q^{2} \frac{1-z}{z}, \mu\right) \frac{x}{z} \phi_{q}^{\mathrm{ns}}\left(\frac{x}{z}, \mu\right)
$$

for the non-singlet part of the structure function $F_{2}\left(x, Q^{2}\right)$. The result (11) is valid in the threshold region at leading power in $M_{X}^{2} / Q^{2} \approx(1-x)$ and $\Lambda_{\mathrm{QCD}}^{2} / M_{X}^{2}$. As long as $M_{X} \gg$ $\Lambda_{\mathrm{QCD}}$, both the jet function $J\left(M_{X}^{2}, \mu\right)$ and the hard function $H\left(Q^{2}, \mu\right)$ can be evaluated in perturbation theory, whereas the parton distribution function $\phi_{q}^{\mathrm{ns}}(\xi, \mu)$ is a non-perturbative object. The result for the hard function involves single and (Sudakov) double logarithms of the form $\alpha_{s}^{n} \ln ^{m}(Q / \mu)$, with $m \leq 2 n$, while the integral over the jet function produces logarithms $\alpha_{s}^{n} \ln ^{m}\left(M_{X} / \mu\right)$. Irrespective of the value of the renormalization scale $\mu$, the fixed-order result contains large logarithms.

Traditionally, the resummation of these logarithms is performed in moment space. The threshold region of small $M_{X}$ is probed by large- $N$ moments. The relevant scale in Mellin space is $Q / \sqrt{N}$, so that the large perturbative logarithms depend on the moment parameter $N$. In 1, 2] it was shown that these logarithms can be absorbed into a resummation exponent $G_{N}$, defined by integrals over two radiation functions $A_{q}\left(\alpha_{s}\right)$ and $B_{q}\left(\alpha_{s}\right)$,

$$
G_{N}\left(Q^{2}, \mu\right)=\int_{0}^{1} d z \frac{z^{N-1}-1}{1-z}\left[\int_{\mu^{2}}^{(1-z) Q^{2}} \frac{d k^{2}}{k^{2}} A_{q}\left(\alpha_{s}(k)\right)+B_{q}\left(\alpha_{s}(Q \sqrt{1-z})\right)\right] .
$$

The functions $A_{q}$ and $B_{q}$ are determined by matching with results from fixed-order perturbation theory and are currently known at three-loop order, enabling a nearly complete threshold resummation to next-to-next-to-next-to-leading logarithmic ( $\mathrm{N}^{3} \mathrm{LL}$ ) accuracy 44. The resummed momentum-space structure function $F_{2}\left(x, Q^{2}\right)$ is obtained from the moment-space expression by an inverse Mellin transformation.

This approach to threshold resummation has several drawbacks. The first is related to integrations over the Landau pole in the running coupling. These occur twice: once in the integrals over the functions $A_{q}$ and $B_{q}$ in the resummation exponent, and once again when the inverse Mellin transform is taken to obtain results in momentum space. To perform the 
resummation one needs to specify a prescription for how to deal with these poles. Various methods have been proposed in the literature, such as the "minimal prescription" [5] or the "tower expansion" [6]. The difference between these prescriptions is a power-suppressed effect. Since factorization theorems do receive power corrections, this does not appear as a problem at first sight. However, as discussed in [7, the Landau-pole singularity in the resummed expression can induce large unphysical power corrections. In the example of the Drell-Yan process, the ambiguity in the threshold resummation amounts to a power correction of order $\Lambda_{\mathrm{QCD}} / M_{X}$, while the physical power corrections to the process scale as $\Lambda_{\mathrm{QCD}}^{2} / M_{X}^{2}$. The fact that resummations with RG methods [7, 8] do not involve Landau-pole ambiguities illustrates that these effects do not have a direct physical interpretation. In particular, a Landau-pole ambiguity does not necessarily imply the presence of a commensurate renormalon ambiguity 7]. Further drawbacks are that in the traditional resummation formalism the separation of contributions from the hard and jet scales is not transparent, and while the function $A_{q}$ has a clear interpretation as the cusp anomalous dimension familiar from the renormalization theory of Wilson lines [9, 10, the function $B_{q}$ is not easily identified with a field-theoretical object.

In this paper we use RG techniques to perform the resummation of perturbative logarithms directly in momentum space. The starting point is the factorization formula (11), which we rederive using soft-collinear effective theory (SCET) 11, 12, 13, 14. In this framework, the hard function $H$ and the jet function $J$ are matching coefficients. The hard function arises from a first matching step, in which the electroweak current is matched onto a corresponding effective-theory current operator. In a second step, the partons associated with the hadronic final state are integrated out, giving rise to the jet function. Threshold logarithms are resummed by solving the $\mathrm{RG}$ equations for these matching functions, using techniques presented in [15]. Existing results from higher-order perturbative calculations enable us to perform the matching and resummation up to next-to-next-to-leading order (NNLO) in RGimproved perturbation theory, corresponding to the $\mathrm{N}^{3} \mathrm{LL}$ approximation in the standard approach. We show that the results obtained in momentum space are formally equivalent to the more familiar moment-space results by deriving a formula which connects them order by order in perturbation theory. However, integrals over the Landau pole never appear in our momentum-space formulation, and the effective-theory matching functions and anomalous dimensions have a clear field-theoretical interpretation. Furthermore, we obtain a simple analytic expression for the resummed structure function, while the Mellin inversion which is necessary in the traditional approach can only be performed numerically. As a result, it is straightforward to match our resummed expressions onto fixed-order calculations valid outside the threshold region. Finally, we stress that our approach to resummation in $x$-space is free of the pathologies related to large unphysical power ambiguities found in [5]. In fact, it exhibits a better apparent perturbative convergence than the conventional approach.

In the context of SCET, the generic factorization formula for DIS has been discussed previously in [16, while the case $x \rightarrow 1$ has been studied in [17, 18, 19, 20, 21, 22]. These papers make conflicting statements about the factorization properties of DIS in the endpoint region. Most of the differences are resolved after observing that, near the endpoint, the parton distribution function receives contributions from two distinct non-perturbative modes. While the two modes cannot be factorized perturbatively, their presence must nonetheless be taken into account to correctly translate the effective-theory result into the QCD factorization 
theorem (11). In [7, 17, 19, 21, 23, the resummation was performed by solving the RG equations for the moments. This avoids Landau-pole singularities in the exponent, but as we show here, it is possible to solve the equations directly in momentum space.

While threshold resummation in DIS is of limited phenomenological importance, it is a relatively simple process for which the perturbative results needed in our calculation are known at NNLO. For this reason, it provides an especially instructive example with which to develop our resummation formalism. However, many other processes fulfill factorization theorems of the same structure, in which the rate factorizes into a hard contribution times a jet function convoluted with a nonperturbative matrix element, and our formalism also applies to these cases. An example is heavy-particle production near threshold, which includes the DrellYan process in the limit where the invariant mass of the produced lepton pair is close to the center-of-mass energy in the collision, as well as Higgs production in the same kinematic region. Another example is provided by inclusive $B$-meson decays in the endpoint region. Our final result for the resummed DIS structure function is very similar to the factorized expression for radiative $B \rightarrow X_{s} \gamma$ decay as derived in [24, 25]. In fact, both processes involve the same jet function, given by the quark propagator in light-cone gauge.

The outline of the paper is as follows. In Section 2 we use SCET to obtain the QCD factorization formula for DIS near the endpoint, providing a translation between the effective theory and standard discussions. In Section 3 we work out the technique for threshold resummation in momentum space, derive a compact expression for the factorized structure function, and give results for the perturbative matching coefficients valid to NNLO in perturbation theory. In Section 4 we convert our results to moment space and show how they are connected to those obtained in the standard approach. The Appendix gives the perturbative expansions of the RG functions used in our analysis.

\section{Factorization in DIS}

In this section we derive the QCD factorization formula for the non-singlet DIS structure function $F_{2}^{\mathrm{ns}}\left(x, Q^{2}\right)$, using the technology of SCET [11, 12, 13, 14. We consider DIS of electrons off a nuclear target, $e^{-}+N(p) \rightarrow e^{-}+X(P)$, as illustrated in Figure 1. All nontrivial hadronic physics is encoded in the hadronic tensor

$$
\begin{aligned}
W^{\mu \nu}(p, q) & =i \int d^{4} x e^{i q \cdot x}\left\langle N(p)\left|T\left\{J^{\dagger \mu}(x) J^{\nu}(0)\right\}\right| N(p)\right\rangle \\
& =\left(\frac{q^{\mu} q^{\nu}}{q^{2}}-g^{\mu \nu}\right) W_{1}+\left(p^{\mu}-q^{\mu} \frac{p \cdot q}{q^{2}}\right)\left(p^{\nu}-q^{\nu} \frac{p \cdot q}{q^{2}}\right) W_{2},
\end{aligned}
$$

averaged over the nucleon spin. Here $J^{\mu}=\bar{\psi} \gamma^{\mu} \psi$ is the electromagnetic current. The scalar functions $W_{i}$ can be expressed in terms of the kinematic invariants

$$
Q^{2}=-q^{2}, \quad x=\frac{Q^{2}}{2 p \cdot q},
$$

where $q=P-p$ is the momentum of the virtual photon, and $x$ is the Bjorken scaling variable. For simplicity we focus on the flavor non-singlet component of the cross section, 


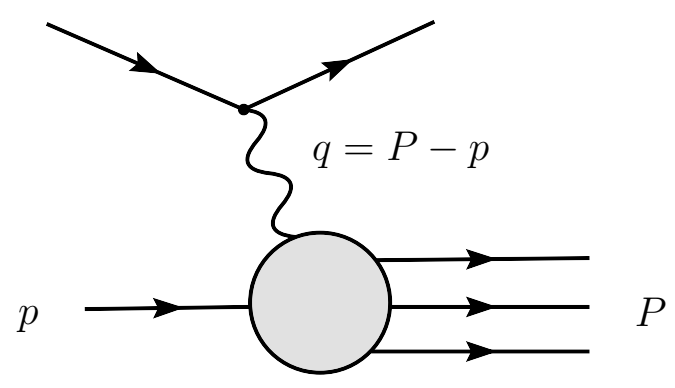

Figure 1: Kinematics of DIS.

which is insensitive to the gluon distribution in the nucleon. It can be obtained by taking the difference of the DIS cross sections for scattering off different target nuclei.

The first step in analyzing the hadronic tensor is to identify which momentum regions give non-vanishing contributions to the Feynman diagrams for $W^{\mu \nu}$ in perturbative QCD. Each region is represented by a set of fields in SCET. The identification of regions can be done in any Lorentz frame. The number of modes and their relative scaling is Lorentz invariant. ${ }^{1}$ Two particularly convenient reference frames are the target rest frame, where $p^{\mu}=(m, 0,0,0)$ with $m$ the nucleon mass, and the Breit frame, where the virtual photon carries momentum $q^{\mu}=(0,0,0, Q)$. The two frames are related to each other by a Lorentz boost along the $z$-direction. Introducing the light-cone decomposition

$$
p^{\mu}=(n \cdot p) \frac{\bar{n}^{\mu}}{2}+(\bar{n} \cdot p) \frac{n^{\mu}}{2}+p_{\perp}^{\mu} \equiv p_{+}^{\mu}+p_{-}^{\mu}+p_{\perp}^{\mu},
$$

where $n^{\mu}=(1,0,0,1)$ and $\bar{n}^{\mu}=(1,0,0,-1)$ are two light-like basis vectors $(n \cdot \bar{n}=2)$, a generic momentum $p_{\text {Lab }}^{\mu}=p_{+}^{\mu}+p_{-}^{\mu}+p_{\perp}^{\mu}$ in the target rest frame transforms into $p_{\text {Breit }}^{\mu}=$ $e^{\eta} p_{+}^{\mu}+e^{-\eta} p_{-}^{\mu}+p_{\perp}^{\mu}$ in the Breit frame, with the rapidity $\eta$ of the boost given by

$$
e^{ \pm \eta}=\frac{Q}{2 m x}\left(\sqrt{1+\frac{4 m^{2} x^{2}}{Q^{2}}} \pm 1\right) .
$$

We shall discuss the different regions in the Breit frame, where the final-state hadronic jet moves along the $z$-direction, while the target nucleon moves in the opposite direction. The light-cone projections of the relevant momenta are (all perpendicular components vanish by choice of coordinates)

$$
\begin{array}{ll}
n \cdot q=-Q, & \bar{n} \cdot q=Q, \\
n \cdot p=m e^{\eta}=\frac{Q}{x}+\frac{m^{2} x}{Q}+\ldots, & \bar{n} \cdot p=m e^{-\eta}=\frac{m^{2} x}{Q}+\ldots, \\
n \cdot P=Q \frac{1-x}{x}+\frac{m^{2} x}{Q}+\ldots, & \bar{n} \cdot P=Q+\frac{m^{2} x}{Q}+\ldots,
\end{array}
$$

\footnotetext{
${ }^{1}$ We disagree with the claim of [17] that fewer momentum regions contribute in the target rest frame than in the Breit frame.
} 
where the neglected terms are of order $m^{4} x^{3} / Q^{3}$. The invariant mass $M_{X}$ of the final-state hadronic jet is given by

$$
M_{X}^{2}=P^{2}=Q^{2} \frac{1-x}{x}+m^{2} \approx Q^{2} \frac{1-x}{x} \gg m^{2} .
$$

In the last step we have used that for an inclusive process the jet mass must be much larger than $m \sim \Lambda_{\mathrm{QCD}}$. Otherwise, the cross section cannot be analyzed using short-distance methods.

While the momentum of the virtual photon is fixed by kinematics, the final-state jet and target nucleon consist of jets of near on-shell partons, whose momenta have scalings consistent with the relations above. We introduce a small expansion parameter $\lambda \sim m / Q \sim \Lambda_{\mathrm{QCD}} / Q$ and quote the components $\left(p_{+}, p_{-}, p_{\perp}\right)$ of parton momenta in units of $Q$. Assume first that $x=\mathcal{O}(1)$ is not very close to 1 . Then, for the purposes of power counting, it follows that $q \sim Q(1,1,0), p \sim Q\left(1, \lambda^{2}, 0\right)$, and $P \sim Q(1,1,0)$. The partons making up the initial and final hadronic states have generic scalings

$$
\begin{array}{lll}
\text { target nucleon: } & p_{\bar{c}} \sim Q\left(1, \lambda^{2}, \lambda\right) & (\text { anti-collinear }), \\
\text { final-state jet: } & p_{h} \sim Q(1,1,1) \quad(\text { hard }),
\end{array}
$$

where the term "anti-collinear" refers to collinear fields propagating in the negative $z$-direction. These relations change for the special case where $x$ is close to 1 , such that $\epsilon=1-x$ becomes parametrically small. The momentum of the final-state jet now scales like $P \sim Q(\epsilon, 1,0)$. While the valence quark in the target nucleon struck by the photon still carries an anticollinear momentum $p_{\text {valence }} \sim Q\left(1, \lambda^{2}, \lambda\right)$, the remaining partons in the nucleon now have total momentum scaling like $p-p_{\text {valence }} \sim Q\left(\epsilon, \lambda^{2}, \lambda\right)$. Consequently, the partons making up the final-state jet and the target remnant jet have momenta scaling like

$$
\begin{array}{rll}
\text { final-state jet: } & p_{h c} \sim Q(\epsilon, 1, \sqrt{\epsilon}) & \text { (hard-collinear) }, \\
\text { target remnants: } & p_{s c} \sim Q\left(\epsilon, \lambda^{2}, \sqrt{\epsilon} \lambda\right) & \text { (soft-collinear) } .
\end{array}
$$

In these relations, the scaling of the perpendicular momentum components follows from the requirement that the individual partons be nearly on-shell. The terminology for the "hardcollinear" and "soft-collinear" modes follows [26] and 27]. In the traditional literature on factorization in DIS [1, 2, 3] the soft-collinear modes were referred to as "soft". Relation (8) implies that $\lambda^{2} \ll \epsilon \ll 1$, and there is no need to specify the relative scaling between $\epsilon$ and $\lambda$ in more detail. All that matters for the factorization analysis is that $p_{h c}^{2} \sim Q^{2} \epsilon$ is a perturbative scale, while $p_{\bar{c}}^{2} \sim Q^{2} \lambda^{2}$ is not.

The discussion of factorization for the generic case, where $x=\mathcal{O}(1)$ but not very close to 1, is straightforward [16]. Hard modes are described by QCD, whereas the anti-collinear partons making up the target nucleon can be described in SCET. There is no need to include any other modes, since the only relevant regions are hard and anti-collinear. In interactions of the anti-collinear fields with hard fields, only the large plus components $p_{\bar{c}+} \sim Q$ of the anti-collinear momenta should be kept at leading order in power counting. Correspondingly, the anti-collinear fields must be multipole expanded about $x_{-}=(\bar{n} \cdot x) n / 2$, i.e., $\phi_{\bar{c}}(x)=$ 
$\phi_{\bar{c}}\left(x_{-}\right)+\ldots$ Integrating out the hard modes by matching onto SCET yields an expression for the discontinuity of the hadronic tensor of the form

$$
\frac{1}{\pi} \operatorname{Im} W^{\mu \nu}=\int_{x}^{1} \frac{d \xi}{x} C\left(Q^{2}, x / \xi, \mu\right) \int \frac{d t}{2 \pi} e^{-i \xi n \cdot p t}\left\langle N(p)\left|\bar{\psi}(t n)[t n, 0] \gamma^{\mu} \frac{\not h}{2} \gamma^{\nu} \psi(0)\right| N(p)\right\rangle,
$$

where $C=\delta(1-x / \xi)+\mathcal{O}\left(\alpha_{s}\right)$ is a matching coefficient in the effective theory, and the object $[t n, 0]$ is a straight Wilson line along the $n$ light-cone. We have used that the SCET Lagrangian for a single collinear sector is equivalent to the QCD Lagrangian [12] in order to replace the SCET fields by the usual QCD fields. The identification of the nucleon matrix element with the QCD parton distribution function is then automatic (see relation (23) below), and one arrives at the standard factorization formula.

The derivation of the factorization formula for $x \rightarrow 1$ is more complicated. It involves a twostep matching procedure similar to that used for inclusive semi-leptonic and radiative $B$ decays in the endpoint region [24, 25, 26, 28. In a first matching step, hard modes are integrated out by matching QCD onto a version of SCET containing hard-collinear, anti-collinear, and softcollinear fields. We will refer to this intermediate effective theory as $\operatorname{SCET}(h c, \bar{c}, s c)$ for short. The matching function associated with this first step is the hard coefficient $C_{V}$. Because the sum of a hard-collinear momentum and an anti-collinear momentum has an invariant mass $\left(p_{h c}+p_{\bar{c}}\right)^{2} \sim Q^{2}$ and must be counted as hard, the intermediate effective Lagrangian does not contain vertices coupling the hard-collinear fields to anti-collinear ones. These fields interact only through the exchange of soft-collinear "messenger" fields. However, the soft-collinear modes can be decoupled from the hard-collinear ones by means of a field redefinition. After this decoupling, it is possible to integrate out the hard-collinear scale by matching onto a low-energy theory $\operatorname{SCET}(\bar{c}, s c)$ involving only anti-collinear and soft-collinear modes. The matching function associated with this step is the jet function $J$. Having integrated out the perturbative modes, the final step is to evaluate the matrix element of the remaining operator defined in the low-energy effective theory. An important part of the factorization analysis is to show that this matrix element is equivalent to the QCD parton distribution function evaluated in the limit $x \rightarrow 1$, as studied e.g. in [1, 3. We will show that this is indeed the case, and that the soft-collinear modes play an important role in this identification.

The appropriate Lagrangian for $\operatorname{SCET}(h c, \bar{c}, s c)$ is a generalization of the effective Lagrangian for collinear and soft-collinear fields derived in [27, 29. It contains hard-collinear quark and gluon fields $\xi_{h c}$ and $A_{h c}$, anti-collinear quark and gluon fields $\xi_{\bar{c}}$ and $A_{\bar{c}}$, and soft-collinear quark and gluon fields $\theta_{s c}$ and $A_{s c}$. The hard-collinear fields move along the $z$-direction, and hence $\not h \xi_{h c}=0$. The anti-collinear and soft-collinear fields move in the opposite direction, so $\not h \xi_{\bar{c}}=0$ and $\not h \theta_{s c}=0$. The two collinear sectors can only interact via soft-collinear exchange, and at leading power only soft-collinear gluons are involved in these interactions. The corresponding effective Lagrangian at leading order in the expansion parameters $\epsilon$ and $\lambda$ is [27, 29]

$$
\begin{aligned}
\mathcal{L}_{\mathrm{SCET}}(y)= & \bar{\xi}_{h c} \frac{\not h}{2}\left[i n \cdot D_{h c}+g n \cdot A_{s c}\left(y_{-}\right)\right] \xi_{h c}-\bar{\xi}_{h c} i \not D_{h c \perp} \frac{\not h}{2} \frac{1}{i \bar{n} \cdot D_{h c}} i \not D_{h c \perp} \xi_{h c} \\
& +\bar{\xi}_{\bar{c}} \frac{\not h}{2}\left[i \bar{n} \cdot D_{\bar{c}}+g \bar{n} \cdot A_{s c}\left(y_{+}\right)\right] \xi_{\bar{c}}-\bar{\xi}_{\bar{c}} i \not D_{\bar{c} \perp} \frac{\not h}{2} \frac{1}{i n \cdot D_{\bar{c}}} i D_{\bar{c} \perp} \xi_{\bar{c}}
\end{aligned}
$$




$$
+ \text { pure glue terms }+ \text { soft-collinear Lagrangian, }
$$

where all fields without position argument are to be evaluated at the point $y$. The effective Lagrangian is invariant under a set of hard-collinear, anti-collinear, and soft-collinear gauge transformations, whose precise form can be found in [27, 30].

An important property of the SCET Lagrangian is that soft-collinear gluons can be decoupled from the hard-collinear and anti-collinear fields through field redefinitions involving Wilson lines [12, 27]. This decoupling is essential for the factorization analysis below. Diagrammatic factorization proofs also rely on the decoupling of "soft" gluons from collinear fields. The underlying physics is that soft gluons couple to collinear partons through eikonal vertices, a feature explicit in the SCET Lagrangian (12).

\subsection{Matching of the current}

The first step in the factorization procedure is to integrate out hard fluctuations by matching QCD onto the intermediate effective theory $\operatorname{SCET}(h c, \bar{c}, s c)$. The kinematic restrictions implied by the limit $x \rightarrow 1$ simplify this first matching step. Since we are dealing with the region of phase space where the final-state jet is hard-collinear, there are no contributions to the hadronic tensor where the anti-collinear partons at points 0 and $x$ are connected by hard gluons. It is therefore sufficient to integrate out hard fluctuations at the level of the electromagnetic current. Time-ordered products of two currents are not needed until the second step.

We match the QCD current $J^{\mu}(x)=\left(\bar{\psi} \gamma^{\mu} \psi\right)(x)$ onto a current in SCET containing a hard-collinear quark and an anti-collinear anti-quark. The form of the resulting operator is dictated by gauge invariance. The appropriate matching relations for the QCD fields are

$$
\psi_{h c}(x) \rightarrow\left(W_{h c}^{\dagger} \xi_{h c}\right)(x), \quad \psi_{\bar{c}}(x) \rightarrow\left(W_{\bar{c}}^{\dagger} \xi_{\bar{c}}\right)\left(x_{-}\right),
$$

where $W_{h c}$ is the hard-collinear Wilson line

$$
W_{h c}(x)=\mathbf{P} \exp \left(i g \int_{-\infty}^{0} d s \bar{n} \cdot A_{h c}(x+s \bar{n})\right)
$$

along the $\bar{n}$-direction, and $W_{\bar{c}}$ is the analogous anti-collinear Wilson line along the $n$-direction. The multipole expansion in (13) requires some explanation. In the hadronic tensor (3), the points 0 and $x$ are connected by a hard-collinear jet propagating through a cloud of softcollinear partons. This implies that the position argument $x$ scales as a hard-collinear quantity,

$x \sim\left(1, \epsilon^{-1}, \epsilon^{-\frac{1}{2}}\right)$. It follows that not all components of the anti-collinear and soft-collinear momenta must be kept in the calculation of Feynman graphs in the effective theory. The minus and perpendicular components of anti-collinear and soft-collinear momenta are much smaller than the corresponding components of hard-collinear momenta and so should be expanded out. On the other hand, the large plus component $n \cdot p \sim Q$ of the target nucleon is canceled by the momentum component $n \cdot q$ of the current and turned into a momentum component of order $\epsilon Q$, which is of the same order as the plus component of a hard-collinear or softcollinear momentum. For this reason, it would be incorrect to set $x_{-}=0$ in the argument of the soft-collinear fields entering the effective current operator, even though this is the correct 
multipole expansion of Lagrangian interactions between soft-collinear and anti-collinear fields [27. Therefore, when matching the current operator onto SCET, one must multipole expand both the anti-collinear and soft-collinear fields about $x_{-}$.

The two expressions in (13) are invariant under hard-collinear and anti-collinear gauge transformations, while under a soft-collinear gauge transformation both composite fields, $W_{h c}^{\dagger} \xi_{h c}$ and $W_{\bar{c}}^{\dagger} \xi_{\bar{c}}$, transform into $U_{s c}\left(x_{-}\right)$times themselves. Thus, at tree level the gaugeinvariant matching relation for the current is

$$
\left(\bar{\psi} \gamma^{\mu} \psi\right)(x) \rightarrow\left(\bar{\xi}_{\bar{c}} W_{\bar{c}}\right)\left(x_{-}\right) \gamma_{\perp}^{\mu}\left(W_{h c}^{\dagger} \xi_{h c}\right)(x)
$$

Only a single Dirac structure is possible for massless quarks. Beyond tree level the matching relation at leading power gets generalized to (see the analogous discussions in [13, 29])

$$
\begin{aligned}
\left(\bar{\psi} \gamma^{\mu} \psi\right)(x) & \rightarrow \int d t \widetilde{C}_{V}(t, n \cdot q, \mu)\left(\bar{\xi}_{\bar{c}} W_{\bar{c}}\right)\left(x_{-}\right) \gamma_{\perp}^{\mu}\left(W_{h c}^{\dagger} \xi_{h c}\right)(x+t \bar{n}) \\
& =C_{V}(-n \cdot q \bar{n} \cdot \boldsymbol{P}, \mu)\left(\bar{\xi}_{\bar{c}} W_{\bar{c}}\right)\left(x_{-}\right) \gamma_{\perp}^{\mu}\left(W_{h c}^{\dagger} \xi_{h c}\right)(x) .
\end{aligned}
$$

In the first line we have used that $\bar{n} \cdot \partial$ derivatives of hard-collinear fields are unsuppressed in SCET power counting, allowing for arbitrary displacements of these fields along the $\bar{n}$ light-cone. In the second line, the object $\boldsymbol{P}$ is the hard-collinear momentum operator, and

the Wilson coefficient $C_{V}$ is the Fourier transform of the position-space Wilson coefficient $\widetilde{C}_{V}$ appearing in the first line. In the case at hand, the relevant components $-n \cdot q \approx \bar{n} \cdot P \approx Q$ are fixed by kinematics (see the relations (17)), and so we may write $C_{V}\left(Q^{2}, \mu\right)$ for simplicity.

\subsection{Matching of the hadronic tensor}

The next step in the matching procedure is to evaluate the hadronic tensor in the intermediate effective theory. Inserting the SCET current (16) into (3), we find the leading-power expression

$$
\begin{aligned}
W^{\mu \nu}(p, q) & \rightarrow\left|C_{V}\left(Q^{2}, \mu\right)\right|^{2} i \int d^{4} x e^{i q \cdot x} \\
& \times\left\langle N(p)\left|T\left\{\left(\bar{\xi}_{\bar{c}} W_{\bar{c}}\right)\left(x_{-}\right) \gamma_{\perp}^{\mu}\left(W_{h c}^{\dagger} \xi_{h c}\right)(x)\left(\bar{\xi}_{h c} W_{h c}\right)(0) \gamma_{\perp}^{\nu}\left(W_{\bar{c}}^{\dagger} \xi_{\bar{c}}\right)(0)\right\}\right| N(p)\right\rangle .
\end{aligned}
$$

The interactions of soft-collinear gluons with hard-collinear fields in (12) can be removed by the field redefinitions [12, 27]

$$
\xi_{h c}(x) \rightarrow S_{n}\left(x_{-}\right) \xi_{h c}^{(0)}(x), \quad A_{h c}^{\mu}(x) \rightarrow S_{n}\left(x_{-}\right) A_{h c}^{\mu(0)}(x) S_{n}^{\dagger}\left(x_{-}\right)
$$

which imply $\left(W_{h c}^{\dagger} \xi_{h c}\right)(x) \rightarrow S_{n}\left(x_{-}\right)\left(W_{h c}^{(0) \dagger} \xi_{h c}^{(0)}\right)(x)$. Here

$$
S_{n}(x)=\mathbf{P} \exp \left(i g \int_{-\infty}^{0} d s n \cdot A_{s c}(x+s n)\right)
$$

is a soft-collinear Wilson line along the $n$-direction. The redefined hard-collinear fields with superscripts "(0)" are decoupled from soft-collinear fields and thus interact only among themselves. After the field redefinition the hadronic matrix element in (17) factorizes into a vacuum 


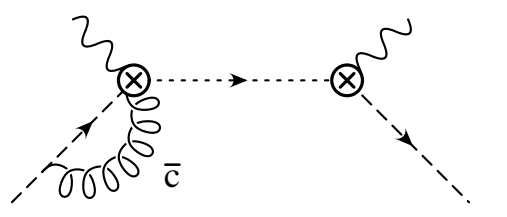

(a)

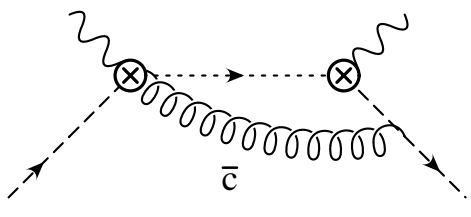

(b)

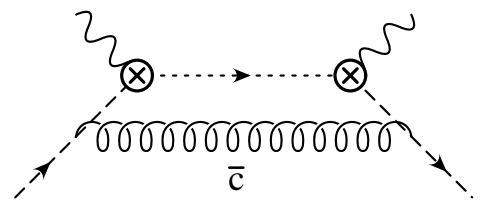

(c)

Figure 2: Examples of diagrams involving anti-collinear gluon exchange. The dashed (dotted) lines represent anti-collinear (hard-collinear) quark lines. The wavy lines represent the electromagnetic currents. In graph (a) the anti-collinear gluon is part of the initial-state (nucleon) jet and the final-state propagator is hard-collinear, as required in the effective theory. In graphs (b) and (c) the anti-collinear gluon is part of the final-state jet, whose invariant mass then becomes hard. Graphs (b) and (c) are therefore not part of the effective-theory representation of the hadronic tensor as $x \rightarrow 1$.

matrix element of hard-collinear fields and a nucleon matrix element of anti-collinear and softcollinear fields. In the second matching step, we "integrate out" the hard-collinear fields, which can be done using perturbation theory because the hard-collinear scale is a short-distance scale, $p_{h c}^{2} \sim Q^{2}(1-x) \gg \Lambda_{\mathrm{QCD}}^{2}$. Since in a single (hard-)collinear sector SCET is equivalent to full QCD [13], the vacuum matrix element of hard-collinear fields can be rewritten in terms of the QCD matrix element 31]

$$
\begin{aligned}
\left\langle 0\left|T\left\{\left(W_{h c}^{(0) \dagger} \xi_{h c}^{(0)}\right)(x)\left(\bar{\xi}_{h c}^{(0)} W_{h c}^{(0)}\right)(0)\right\}\right| 0\right\rangle & =\left\langle 0\left|T\left[\frac{\not h \hbar}{4} W^{\dagger}(x) \psi(x) \bar{\psi}(0) W(0) \frac{\not h h}{4}\right]\right| 0\right\rangle \\
& =\int \frac{d^{4} k}{(2 \pi)^{4}} e^{-i k \cdot x} \frac{\not h}{2} \bar{n} \cdot k \mathcal{J}\left(k^{2}, \mu\right) .
\end{aligned}
$$

The object $W(x)$ denotes a Wilson line analogous to (14) but with gauge fields in full QCD. Color indices are suppressed; the correlator is proportional to the unit matrix in color space. We define the jet function through the imaginary part of $\mathcal{J}$ as (see e.g. [32])

$$
J\left(p^{2}, \mu\right)=\frac{1}{\pi} \operatorname{Im}\left[i \mathcal{J}\left(p^{2}, \mu\right)\right] .
$$

The jet function has support for $p^{2}>0$.

At this point it is important to emphasize a subtlety related to the matching of the forwardscattering amplitude in full QCD onto operator matrix elements in SCET. The anti-collinear composite fields $W_{\bar{c}}^{\dagger} \xi_{\bar{c}}$ and $\bar{\xi}_{\bar{c}} W_{\bar{c}}$ in (17) are not allowed to communicate via anti-collinear particle exchanges, but only through exchanges of soft-collinear partons. The exchange of anticollinear particles between the two currents in (33) is kinematically forbidden in the region $x \rightarrow$ 1 , as this would lead to a final-state invariant hadronic mass $M_{X} \sim Q$. Since the intermediate state is hard instead of hard-collinear, diagrams such as those shown in Figure 2(b) and (c) are not part of the effective-theory representation of the hadronic tensor in the region $x \rightarrow 1$. Such "forbidden" graphs are nonetheless generated (and yield non-vanishing results) 
if the SCET Feynman rules used for the matching of the electromagnetic current are naively applied to the hadronic tensor. We can construct a set of Feynman rules appropriate for the hadronic tensor by introducing different anti-collinear fields for the "in" and "out" states in the forward-scattering amplitude, and restricting interactions between the two anti-collinear sectors to soft-collinear exchange. These effective-theory Feynman rules produce graphs such as that in Figure 2(a), but not those in Figure 2(b) and (c). For simplicity of notation, we will suppress the "in" and "out" labels on the anti-collinear fields, but one must make this distinction when evaluating the hadronic tensor in the effective theory.

After integrating out the hard-collinear fields, the resulting nucleon matrix element in the low-energy effective theory can be reduced to

$$
\begin{aligned}
& \left\langle N(p)\left|\left(\bar{\xi}_{\bar{c}} W_{\bar{c}}\right)\left(x_{-}\right) S_{n}\left(x_{-}\right) \gamma_{\perp}^{\mu} \frac{\not h}{2} \gamma_{\perp}^{\nu} S_{n}^{\dagger}(0)\left(W_{\bar{c}}^{\dagger} \xi_{\bar{c}}\right)(0)\right| N(p)\right\rangle \\
= & -\left\langle N(p)\left|\left(\bar{\xi}_{\bar{c}} W_{\bar{c}}\right)\left(x_{-}\right)\left[x_{-}, 0\right]_{s c}\left(g_{\perp}^{\mu \nu}-i \epsilon_{\perp}^{\mu \nu} \gamma_{5}\right) \frac{\not h}{2}\left(W_{\bar{c}}^{\dagger} \xi_{\bar{c}}\right)(0)\right| N(p)\right\rangle,
\end{aligned}
$$

where $\left[x_{-}, 0\right]_{s c}=S_{n}\left(x_{-}\right) S_{n}^{\dagger}(0)$ is a straight Wilson line of soft-collinear gluon fields along the $n$ light-cone. In the second line we have defined the objects $g_{\perp}^{\mu \nu}=g^{\mu \nu}-\frac{1}{2}\left(n^{\mu} \bar{n}^{\nu}+\bar{n}^{\mu} n^{\nu}\right)$ and $\epsilon_{\perp}^{\mu \nu}=\frac{1}{2} \epsilon^{\mu \nu \alpha \beta} \bar{n}_{\alpha} n_{\beta}$, and also used that $\not h \xi_{\bar{c}}=0$. The anti-symmetric structure vanishes after averaging over the nucleon spin. The appearance of the symmetric structure $g_{\perp}^{\mu \nu}$ implies the Callan-Gross relation $Q^{2} W_{2}=4 x^{2} W_{1}$ at leading power and to all orders in perturbation theory. Hereafter, we thus focus on the structure function $W_{1}$.

Consider now the standard definition of the quark distribution function in QCD [33],

$$
\phi_{q}^{\mathrm{ns}}(\xi, \mu)=\frac{1}{2 \pi} \int_{-\infty}^{\infty} d t e^{-i \xi t n \cdot p}\left\langle N(p)\left|\bar{\psi}(t n)[t n, 0] \frac{\not h}{2} \psi(0)\right| N(p)\right\rangle
$$

where $[t n, 0]$ is a straight Wilson line of gauge fields in full QCD, and the superscript "ns" indicates the flavor non-singlet component of the distribution function. In the Breit frame, where the proton moves along the $\bar{n}$-direction, $\psi$ and $\bar{\psi}$ can be considered anti-collinear fields. For generic values of $\xi$ these fields carry only a portion of the proton's longitudinal momentum. The remaining portion $(1-\xi) n \cdot p$ is still large and can be shared between other anti-collinear partons exchanged between the two points 0 and $t n$. A different picture is called for in the limit $\xi \rightarrow 1$, where the anti-collinear valence quarks $\psi$ and $\bar{\psi}$ carry almost all of the longitudinal momentum [3]. In this case, the residual momentum component $(1-\xi) n \cdot p$ is small, and the remaining partons are soft-collinear. Each valence quark is described by an anti-collinear jet propagating through the soft-collinear cloud made up of the remaining partons. The two anti-collinear jets communicate through soft-collinear gluon exchange only.

The distinct roles played by the valence and remaining partons as $\xi \rightarrow 1$ make it appropriate to introduce an effective field-theory description for the parton distribution function, in which it is matched onto an operator involving anti-collinear and soft-collinear fields in SCET. The most general, gauge-invariant form the relation (23) can be matched onto in the $\xi \rightarrow 1$ limit reads

$$
\left.\phi_{q}^{\mathrm{ns}}(\xi, \mu)\right|_{\xi \rightarrow 1}=\frac{1}{2 \pi} \int_{-\infty}^{\infty} d t e^{-i \xi t n \cdot p}\left\langle N(p)\left|\left(\bar{\xi}_{\bar{c}} W_{\bar{c}}\right)(t n)[t n, 0]_{s c} \frac{\not h}{2}\left(W_{\bar{c}}^{\dagger} \xi_{\bar{c}}\right)(0)\right| N(p)\right\rangle .
$$


It is understood that the anti-collinear fields located at the points 0 and $t n$ interact only via soft-collinear gluon exchange. Both (24) and the QCD matrix element (23) depend on the single invariant $p^{2}=m^{2}$, so there is no non-trivial hard matching coefficient. The matrix element (24) is precisely the object we encountered in (22). We can use this correspondence along with some simple algebra to find

$$
W_{1}=\left|C_{V}\left(Q^{2}, \mu\right)\right|^{2} i \int d(n \cdot k) \bar{n} \cdot q \mathcal{J}\left(q^{2}+n \cdot k \bar{n} \cdot q, \mu\right) \phi_{q}^{\mathrm{ns}}\left(\frac{n \cdot k}{n \cdot p}, \mu\right) .
$$

The structure function $F_{2}^{\mathrm{ns}}\left(x, Q^{2}\right)$ equals $\sum_{q} e_{q}^{2} x \frac{1}{\pi} \operatorname{Im} W_{1}$, where the $e_{q}$ are quark electric charges. Inserting the definition of the jet function (21), and recalling that $q^{2}=-Q^{2}$ and $n \cdot p \bar{n} \cdot q=Q^{2} / x+$ power corrections, we obtain the final result for the factorization formula

$$
F_{2}^{\mathrm{ns}}\left(x, Q^{2}\right)=\sum_{q} e_{q}^{2}\left|C_{V}\left(Q^{2}, \mu\right)\right|^{2} Q^{2} \int_{x}^{1} d \xi J\left(Q^{2} \frac{\xi-x}{x}, \mu\right) \phi_{q}^{\mathrm{ns}}(\xi, \mu) .
$$

This formula is valid to all orders in perturbation theory and at leading power in $(1-x)$ and $\Lambda_{\mathrm{QCD}}^{2} / M_{X}^{2}$. The argument of the jet function takes values between 0 and $M_{X}^{2}$, where the total jet invariant mass was given in (8). The equivalent form (11) is obtained by substituting $\xi=$ $x / z$. At tree-level, this formula evaluates to the familiar parton-model expression $F_{2}^{\mathrm{ns}}\left(x, Q^{2}\right)=$ $\sum_{q} e_{q}^{2} x \phi_{q}^{\text {ns }}(x)$.

Relation (26) is the standard form of the QCD factorization formula for the DIS structure function in the limit $x \rightarrow 1$ [1, 2, 3, which we have derived here using SCET. We hope our derivation helps resolve some of the disagreements in the literature. Soft-collinear messenger modes obviously play a crucial role in the derivation, as the parton distribution function at large $\xi$ is defined in terms of these fields. The proper effective-theory description of the parton distribution function thus requires two distinct non-perturbative modes. This element is missing from [17, 20, where it was argued that only one non-perturbative mode is needed, either because the soft graphs vanish in the Breit frame calculation, or because the effectivetheory formulation in the target rest frame involves only one non-perturbative mode from the beginning. Although we disagree with these statements (the second of which would violate reparameterization invariance in the effective theory), our explicit one-loop results agree with those derived in these papers. This is because our findings imply that parton evolution in the endpoint region can be described simply by taking the $x \rightarrow 1$ limit of the Altarelli-Parisi splitting functions, which is effectively what was done in the calculations of [17]. Our explicit one-loop results also agree with those in [18, where the power counting $\epsilon=1-x \sim \lambda=$ $\Lambda_{\mathrm{QCD}} / Q$ was adopted. While this counting is possible and natural in view of the hierarchy $\lambda^{2} \ll \epsilon \ll 1$, it does not imply that the soft-collinear scale $m^{2}(1-x)$ depends on the scale $Q$, and the presence of this scale does not translate into non-perturbative $Q$-dependence in the parton distribution function, as was suggested in 18. Finally, we have shown that the soft-collinear contributions are precisely such that they can be absorbed into the parton distribution function. We therefore do not confirm the claims of soft contributions outside the parton distribution function made in [19]. The same conclusion as ours was reached in [21, 22, where it was argued that the infrared divergences due to collinear and soft emissions can be absorbed in the standard QCD parton distribution function, although [21] did not discuss 


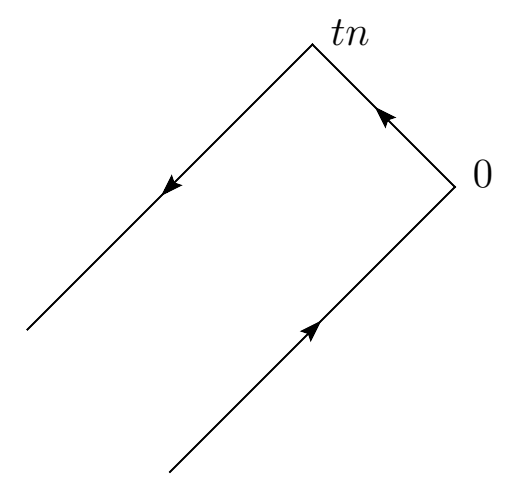

Figure 3: Soft-collinear Wilson line $W_{C}$.

how to obtain this result in the effective theory. In [22] it was claimed that there is a doublecounting problem in SCET, which must be remedied by making certain soft subtractions from the collinear matrix element. We have shown here that there is no such problem. Double counting occurs only if one fails to notice that collinear emissions such as those shown in Figure 2(b) and (c) must not be included in the effective-theory calculation near the endpoint. Similar to [17, the discussion in 22] fails to distinguish the virtualities of hard-collinear and anti-collinear modes, and it overlooks the fact that the smallest scale in the problem is not $Q^{2}(1-x)^{2}$ but $m^{2}(1-x)$.

In the next subsection, we will emphasize the importance of soft-collinear Wilson loops in determining the RG properties of the effective theory.

\subsection{Decoupling transformation and cusp singularities}

An important step in the derivation of the factorization formula (26) was the identification of the parton distribution function for $\xi \rightarrow 1$ with the SCET matrix element on the right-hand side of (24). We can simplify this relation further by decoupling the soft-collinear gluons from the anti-collinear fields with the help of the field redefinitions

$$
\xi_{\bar{c}}(y) \rightarrow S_{\bar{n}}\left(y_{+}\right) \xi_{\bar{c}}^{(0)}(y), \quad A_{\bar{c}}^{\mu}(y) \rightarrow S_{\bar{n}}\left(y_{+}\right) A_{\bar{c}}^{\mu(0)}(y) S_{\bar{n}}^{\dagger}\left(y_{+}\right),
$$

where the soft-collinear Wilson line $S_{\bar{n}}$ is defined in analogy with (19), but with $n$ replaced by $\bar{n}$. Above, $y$ is a generic argument of a term in the SCET Lagrangian. The multipole expansion of the soft-collinear fields about $y_{+}$must be done everywhere except at the location of the current, where $x$ scales as a hard-collinear (not anti-collinear) quantity, see above. At this one point, we have instead

$$
\xi_{\bar{c}}\left(x_{-}\right) \rightarrow S_{\bar{n}}\left(x_{-}\right) \xi_{\bar{c}}^{(0)}\left(x_{-}\right), \quad A_{\bar{c}}^{\mu}\left(x_{-}\right) \rightarrow S_{\bar{n}}\left(x_{-}\right) A_{\bar{c}}^{\mu(0)}\left(x_{-}\right) S_{\bar{n}}^{\dagger}\left(x_{-}\right) .
$$

It follows that

$$
\left.\phi_{q}^{\mathrm{ns}}(\xi, \mu)\right|_{\xi \rightarrow 1}=\frac{1}{2 \pi} \int_{-\infty}^{\infty} d t e^{-i \xi t n \cdot p}\left\langle N(p)\left|\left(\bar{\xi}_{\bar{c}}^{(0)} W_{\bar{c}}^{(0)}\right)(t n) \frac{\not h}{2} W_{C}(t)\left(W_{\bar{c}}^{(0) \dagger} \xi_{\bar{c}}^{(0)}\right)(0)\right| N(p)\right\rangle,
$$

where

$$
W_{C}(t)=\left\langle 0\left|S_{\bar{n}}^{\dagger}(t n)[t n, 0]_{s c} S_{\bar{n}}(0)\right| 0\right\rangle=\left\langle 0\left|S_{\bar{n}}^{\dagger}(t n) S_{n}(t n) S_{n}^{\dagger}(0) S_{\bar{n}}(0)\right| 0\right\rangle
$$


describes a closed Wilson loop consisting of the junction of a Wilson line extending from $-\infty$ to 0 along the $\bar{n}$-direction, a finite-length segment from 0 to tn along the $n$-direction, and another Wilson line from $t n$ to $-\infty$ along the $\bar{n}$-direction, see Figure 3 . Anti-collinear virtual particles can be exchanged inside the brackets $\left(W_{\bar{c}}^{(0) \dagger} \xi_{\bar{c}}^{(0)}\right)$ and $\left(\bar{\xi}_{\bar{c}}^{(0)} W_{\bar{c}}^{(0)}\right)$ in (29) but not between them, see Figure 2. These exchanges give rise to non-perturbative renormalization factors $Z^{\frac{1}{2}}(m, \mu) u(p)$, where $u(p)$ is an on-shell spinor, and the only invariant is $p^{2}=m^{2}$. The above formula then reduces to

$$
\left.\phi_{q}^{\mathrm{ns}}(\xi, \mu)\right|_{\xi \rightarrow 1}=Z(m, \mu) \frac{n \cdot p}{2 \pi} \int_{-\infty}^{\infty} d t e^{-i \xi t n \cdot p} W_{C}(t)
$$

This form of the parton distribution function for $\xi \rightarrow 1$ coincides with eq. (2.12) of [3] (where our factor $Z$ is called $H$ ). Performing the same decoupling transformation (28) on the SCET current (16) yields

$$
\left(\bar{\psi} \gamma^{\mu} \psi\right)(x) \rightarrow C_{V}\left(Q^{2}, \mu\right)\left(\bar{\xi}_{\bar{c}}^{(0)} W_{\bar{c}}^{(0)}\right)\left(x_{-}\right) S_{\bar{n}}^{\dagger}\left(x_{-}\right) S_{n}\left(x_{-}\right) \gamma^{\mu}\left(W_{h c}^{(0) \dagger} \xi_{h c}^{(0)}\right)(x) .
$$

Once again, the soft-collinear fields reside in a closed Wilson loop, this time extending from $-\infty$ to the point $x_{-}$along the $n$-direction, and returning to $-\infty$ along the $\bar{n}$-direction.

The appearance of soft-collinear Wilson loops in the SCET representation of the parton distribution function (31) and the electromagnetic current (32) determines their RG properties. In both cases, ultraviolet singularities related to Sudakov double logarithms are governed by the so-called cusp anomalous dimension, $\Gamma_{\text {cusp }}$, which is a universal quantity of perturbative QCD 3, 9, 10. We will confirm this structure in our explicit calculations below.

\subsection{Power corrections}

Our focus in this paper is on the leading-order factorization formula (26), but it is important to keep in mind that this result receives power corrections in the small parameters $\epsilon$ and $\lambda$. A discussion of power corrections using SCET has been carried out for the closely related case of semi-leptonic inclusive $B$ decay in the endpoint region, where it was found that the power corrections factorize order by order in $1 / m_{b}$ 34, 35, 36]. It is evident that the same procedure applies here, so the effective theory offers a systematic tool to calculate power corrections for DIS near the endpoint.

The procedure involves the two-step matching used in the leading-order case. A systematic treatment of the dominant power corrections requires to match the SCET Lagrangian and electromagnetic current at subleading order in $\epsilon$ and $\lambda$. We will not perform this complete matching here, but instead limit ourselves to a qualitative discussion of the two types of power corrections: those to the jet function, which can be calculated perturbatively, and those to the parton distribution function, which cannot. We give examples of each, and explain how to obtain them with effective field-theory techniques.

Corrections to the jet function first appear at order $(1-x) \alpha_{s}\left(\mu_{i}\right)$ and can be calculated perturbatively. To identify the full set of such corrections, one must match the electromagnetic current onto SCET up to order $\epsilon$. Time-ordered products of the subleading SCET currents containing extra hard-collinear fields compared to the leading-order result (16) build up a set of power-suppressed jet functions convoluted with the leading-order parton distribution. 


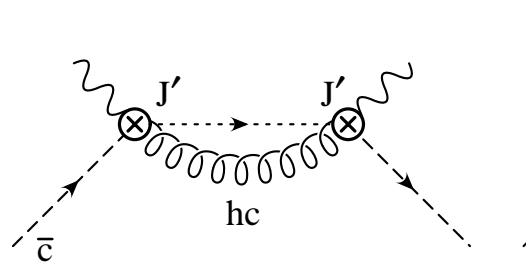

(a)

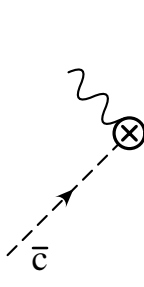

(b)

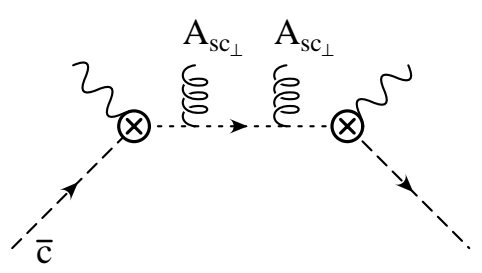

(c)

Figure 4: Examples of subleading time-ordered products in SCET which give rise to power corrections. Graph (a) leads to a perturbatively calculable power-suppressed jet function, while (b) and (c) lead to subleading parton distribution functions depending on three lightcone variables.

As an example, consider the time-ordered product $J^{\prime \dagger}(x) J^{\prime}(0)$ involving two insertions of the $\mathcal{O}(\sqrt{\epsilon})$ suppressed current $J^{\prime}=\bar{n}^{\mu} \bar{\xi}_{\bar{c}} W_{\bar{c}} W_{h c}^{\dagger} i D_{h c \perp} \xi_{h c}$. The relevant one-loop diagram is shown in Figure 4(a). Decoupling the soft-collinear fields and factorizing them into the nucleon matrix element leaves the following vacuum matrix element of hard-collinear fields

$$
\left\langle 0\left|T\left\{\left(W_{h c}^{(0) \dagger} D_{h c \perp}^{(0)} \xi_{h c}^{(0)}\right)(x)\left(\bar{\xi}_{h c}^{(0)} \overleftarrow{D}_{h c \perp}^{(0)} W_{h c}^{(0)}\right)(0)\right\}\right| 0\right\rangle .
$$

The discontinuity of the Fourier-transformed matrix element defines a subleading jet function scaling as $(1-x) \alpha_{s}\left(\mu_{i}\right)$. As in the case of the leading-order jet function, one could equally well calculate the matrix element in full QCD. This particular subleading jet function has been discussed previously in terms of a "non-local OPE" very close in spirit to SCET in [37], and was reconsidered in the context of SCET more recently in [19].

Non-perturbative power corrections are given in terms of a basis of subleading parton distribution functions. These are defined through nucleon matrix elements of power-suppressed $\operatorname{SCET}(\bar{c}, s c)$ operators involving extra anti-collinear and soft-collinear fields compared to the leading-order matrix element (23). To identify the complete basis, we would need to match both the current and the Lagrangians $\mathcal{L}_{\bar{c}+s c}$ and $\mathcal{L}_{h c+s c}$ to subleading order. An important simplification can be made by absorbing all time-ordered products involving insertions of the subleading Lagrangian terms $\mathcal{L}_{\bar{c}+s c}$ into a redefinition of the leading-order parton distribution function $\phi(\xi)$. This can always be done, because such subleading parton distribution functions can only depend on a single light-cone variable $\xi$ and are convoluted with the same jet function. On a more formal level, it amounts to not treating power-suppressed $\mathcal{L}_{\bar{c}+s c}$ terms in the interaction picture, as is normally done. Identifying the remaining non-perturbative structure then reduces to calculating power-suppressed time-ordered products involving subleading SCET currents or insertions of $\mathcal{L}_{h c+s c}$.

The dominant subleading parton distribution function is related to the time-ordered product of the power-suppressed current arising from the multipole expansion of the anti-collinear fields, according to $\left(W_{\bar{c}}^{\dagger} \xi_{\bar{c}}\right)(x)=\left[1+x_{\perp} \cdot \partial_{\perp}+\frac{1}{2}\left(x_{\perp} \cdot \partial_{\perp}\right)^{2}+\ldots\right]\left(W_{\bar{c}}^{\dagger} \xi_{\bar{c}}\right)\left(x_{-}\right)$. Since $x$ is a hard-collinear quantity, $x_{\perp} \cdot \partial_{\perp} \phi_{\bar{c}} \sim(\lambda / \sqrt{\epsilon}) \phi_{\bar{c}}$. Thus the time-ordered product of $J^{\prime \prime \dagger}(x) J(0)$, with

$$
J^{\prime \prime}(x)=\frac{1}{2}\left(\bar{\xi}_{\bar{c}} W_{\bar{c}}\right)\left(x_{-}\right)\left(\overleftarrow{\partial} \cdot x_{\perp}\right)^{2}\left(W_{h c}^{\dagger} \xi_{h c}\right)(x)
$$


results in a power correction of order $\lambda^{2} / \epsilon$. Because the power suppression comes from the current, the associated subleading parton distribution function depends only on a single lightcone variable, as was the case at leading order.

Also interesting is the new multi-local structure related to interactions of soft-collinear fields with the jet. As an example, consider the time-ordered product

$$
J^{(0) \dagger}(x) J^{(0)}(0) \mathcal{L}_{h c+s c}^{\prime}(y) \mathcal{L}_{h c+s c}^{\prime}(z),
$$

involving two insertions of the $\mathcal{O}(\lambda)$ suppressed Lagrangian term $\mathcal{L}_{h c+s c}^{\prime} \sim \bar{\theta}_{s c} A_{h c \perp} \xi_{h c}$. The relevant tree-level Feynman diagram is shown in Figure 4(b). Performing the decoupling transformation and factorizing the soft-collinear fields leads to a tetra-local nucleon matrix element. An additional tetra-local correction of the same order is related to the emission of two transverse soft-collinear gluons, through the Lagrangian term $\mathcal{L}_{h c+s c}^{\prime \prime} \sim \bar{\xi}_{h c} A_{s c \perp} \xi_{h c}$, as illustrated in Figure 4(c). The parton distributions $\phi_{i}\left(\xi_{1}, \xi_{2}, \xi_{3}\right)$ defined by the scalar decomposition of the Fourier-transformed matrix elements appear in a three-fold convolution with a perturbatively calculable jet function, to be treated as power corrections of order $\lambda^{2} \alpha_{s}(\mu)$. For DIS, such multi-local hadronic structures have not yet been considered in the literature.

\section{Renormalization-group evolution and resummation}

The factorization formula for the DIS structure function derived in the previous section contains physics associated with different momentum scales factorized into a hard coefficient function $C_{V}$, a jet function $J$, and a non-perturbative parton distribution function $\phi_{q}^{\text {ns }}$. These three objects depend on a scale $\mu$ at which the corresponding effective-theory operators are renormalized. The hard matching coefficient and the jet function need to be calculated using perturbative QCD. These calculations can be done at fixed order only when the scale is chosen so as to avoid large logarithms: the function $C_{V}$ should be computed at a hard scale $\mu_{h} \sim Q$, while the jet function should be computed at an intermediate scale $\mu_{i} \sim M_{X} \sim Q \sqrt{1-x}$. The results of these calculations must then be evolved to the common scale $\mu$ in (26) by solving $R G$ evolution equations. An advantage of the effective-theory formulation is that the $R G$ equations can be solved directly in momentum space. The method has recently been outlined in [15]; in this section we give a more detailed derivation, filling in the technical steps.

\subsection{Evolution of the hard function}

We begin by discussing the evolution of the hard matching coefficient $C_{V}$ in (16). At leading power there is a single gauge-invariant SCET operator the QCD current can match onto, and hence there is no operator mixing. The exact evolution equation takes the form

$$
\frac{d}{d \ln \mu} C_{V}\left(Q^{2}, \mu\right)=\left[\Gamma_{\text {cusp }}\left(\alpha_{s}\right) \ln \frac{Q^{2}}{\mu^{2}}+\gamma^{V}\left(\alpha_{s}\right)\right] C_{V}\left(Q^{2}, \mu\right),
$$

The appearance of the cusp logarithm and its coefficient can be explained starting from (32) using arguments presented in [29]. This term in the evolution equation is associated with Sudakov double logarithms. The remaining term, $\gamma^{V}$, accounts for single-logarithmic evolution. 
The exact solution to the evolution equation (36) is

$$
C_{V}\left(Q^{2}, \mu\right)=\exp \left[2 S\left(\mu_{h}, \mu\right)-a_{\gamma}\left(\mu_{h}, \mu\right)\right]\left(\frac{Q^{2}}{\mu_{h}^{2}}\right)^{-a_{\Gamma}\left(\mu_{h}, \mu\right)} C_{V}\left(Q^{2}, \mu_{h}\right),
$$

where $\mu_{h} \sim Q$ is a hard matching scale at which the Wilson coefficient $C_{V}$ is calculated using fixed-order perturbation theory. The Sudakov exponent $S$ and the exponents $a_{n}$ are the solutions to the differential equations

$$
\frac{d}{d \ln \mu} S(\nu, \mu)=-\Gamma_{\text {cusp }}\left(\alpha_{s}(\mu)\right) \ln \frac{\mu}{\nu}, \quad \frac{d}{d \ln \mu} a_{\Gamma}(\nu, \mu)=-\Gamma_{\text {cusp }}\left(\alpha_{s}(\mu)\right),
$$

and similarly for $a_{\gamma^{V}}$, subject to the initial conditions $S(\nu, \nu)=a_{\Gamma}(\nu, \nu)=a_{\gamma^{V}}(\nu, \nu)=0$ at $\mu=\nu$. These equations can be integrated by writing $d / d \ln \mu=\beta\left(\alpha_{s}\right) d / d \alpha_{s}$, where $\beta\left(\alpha_{s}\right)=d \alpha_{s} / d \ln \mu$ is the QCD $\beta$-function. This yields the exact solutions [24, 26]

$$
S(\nu, \mu)=-\int_{\alpha_{s}(\nu)}^{\alpha_{s}(\mu)} d \alpha \frac{\Gamma_{\mathrm{cusp}}(\alpha)}{\beta(\alpha)} \int_{\alpha_{s}(\nu)}^{\alpha} \frac{d \alpha^{\prime}}{\beta\left(\alpha^{\prime}\right)}, \quad a_{\Gamma}(\nu, \mu)=-\int_{\alpha_{s}(\nu)}^{\alpha_{s}(\mu)} d \alpha \frac{\Gamma_{\mathrm{cusp}}(\alpha)}{\beta(\alpha)},
$$

and similarly for the function $a_{\gamma^{V}}$. The perturbative expansions of the anomalous dimensions and the resulting expressions for the evolution functions valid at NNLO in RG-improved perturbation theory are given in the Appendix.

\subsection{Evolution of the jet function}

The RG evolution of the jet function is more complicated. It was recently shown that the exact integro-differential evolution equation obeyed by the function $J\left(p^{2}, \mu\right)$ is [31]

$$
\frac{d J\left(p^{2}, \mu\right)}{d \ln \mu}=-\left[2 \Gamma_{\text {cusp }}\left(\alpha_{s}\right) \ln \frac{p^{2}}{\mu^{2}}+2 \gamma^{J}\left(\alpha_{s}\right)\right] J\left(p^{2}, \mu\right)-2 \Gamma_{\text {cusp }}\left(\alpha_{s}\right) \int_{0}^{p^{2}} d p^{\prime 2} \frac{J\left(p^{\prime 2}, \mu\right)-J\left(p^{2}, \mu\right)}{p^{2}-p^{\prime 2}} .
$$

We encounter again the cusp anomalous dimension, and in addition a new function $\gamma^{J}$, which has been calculated at two-loop order in 31.

An important object in the derivation of the solution to this equation is the associated jet function $\widetilde{j}(L, \mu)$, where $L=\ln \left(Q^{2} / \mu^{2}\right)$. This function has originally been defined in terms of an integral over the jet function followed by a certain replacement rule [25]. More elegantly, the associated jet function can be obtained from $J$ by the Laplace transformation

$$
\widetilde{j}\left(\ln \frac{Q^{2}}{\mu^{2}}, \mu\right)=\int_{0}^{\infty} d p^{2} e^{-s p^{2}} J\left(p^{2}, \mu\right), \quad s=\frac{1}{e^{\gamma_{E}} Q^{2}} .
$$

The inverse transformation is

$$
J\left(p^{2}, \mu\right)=\frac{1}{2 \pi i} \int_{c-i \infty}^{c+i \infty} d s e^{s p^{2}} \tilde{j}\left(\ln \frac{1}{e^{\gamma_{E}} s \mu^{2}}, \mu\right)
$$


where the contour must be chosen to stay to the right of all discontinuities (i.e., $c>0$ ). Using the evolution equation (40) for the jet function, we find that the associated jet function obeys the $R G$ equation

$$
\frac{d}{d \ln \mu} \tilde{j}\left(\ln \frac{Q^{2}}{\mu^{2}}, \mu\right)=-\left[2 \Gamma_{\text {cusp }}\left(\alpha_{s}\right) \ln \frac{Q^{2}}{\mu^{2}}+2 \gamma^{J}\left(\alpha_{s}\right)\right] \tilde{j}\left(\ln \frac{Q^{2}}{\mu^{2}}, \mu\right),
$$

which is local in $Q^{2}$ and analogous to the evolution equation (36) for the hard function. The solution to this equation reads

$$
\widetilde{j}\left(\ln \frac{Q^{2}}{\mu^{2}}, \mu\right)=\exp \left[-4 S\left(\mu_{i}, \mu\right)+2 a_{\gamma^{J}}\left(\mu_{i}, \mu\right)\right]\left(\frac{Q^{2}}{\mu_{i}^{2}}\right)^{2 a_{\Gamma}\left(\mu_{i}, \mu\right)} \widetilde{j}\left(\ln \frac{Q^{2}}{\mu_{i}^{2}}, \mu_{i}\right),
$$

where $a_{\gamma^{J}}$ is defined in analogy with (38). Given this solution one can readily derive the solution to the complicated evolution equation (40) for the original jet function by using the inverse transformation (42). The result is

$$
J\left(p^{2}, \mu\right)=\exp \left[-4 S\left(\mu_{i}, \mu\right)+2 a_{\gamma^{J}}\left(\mu_{i}, \mu\right)\right] \frac{e^{-\gamma_{E} \eta}}{\Gamma(\eta)} \int_{0}^{p^{2}} d p^{2} \frac{J\left(p^{\prime 2}, \mu_{i}\right)}{\left(\mu_{i}^{2}\right)^{\eta}\left(p^{2}-p^{\prime 2}\right)^{1-\eta}},
$$

where $\eta=2 a_{\Gamma}\left(\mu_{i}, \mu\right)$. This solution is valid as long as $\eta>0$, which implies that $\mu<\mu_{i}$. Equation (45) is analogous to the solution for the evolution equation of the $B$-meson shape function found in [24, 32] using a technique developed in 38.

Using the connection between $J$ and $\widetilde{j}$ implied by Laplace transformation, it is possible to derive an even more elegant expression for the jet function $J\left(p^{2}, \mu\right)$, which does not involve an integral and which is valid for both $\mu>\mu_{i}$ and $\mu<\mu_{i}$. The result relates $J$ to the associated jet function $\tilde{j}$ evaluated at the scale $\mu_{i}$, where it can be computed using fixed-order perturbation theory. We obtain

$$
J\left(p^{2}, \mu\right)=\exp \left[-4 S\left(\mu_{i}, \mu\right)+2 a_{\gamma^{J}}\left(\mu_{i}, \mu\right)\right] \widetilde{j}\left(\partial_{\eta}, \mu_{i}\right)\left[\frac{1}{p^{2}}\left(\frac{p^{2}}{\mu_{i}^{2}}\right)^{\eta}\right]_{*} \frac{e^{-\gamma_{E} \eta}}{\Gamma(\eta)},
$$

where $\partial_{\eta}$ denotes a derivative with respect to the quantity $\eta$. The star distribution is defined as [32, 39]

$$
\int_{0}^{Q^{2}} d p^{2}\left[\frac{1}{p^{2}}\left(\frac{p^{2}}{\mu^{2}}\right)^{\eta}\right]_{*} f\left(p^{2}\right)=\int_{0}^{Q^{2}} d p^{2} \frac{f\left(p^{2}\right)-f(0)}{p^{2}}\left(\frac{p^{2}}{\mu^{2}}\right)^{\eta}+\frac{f(0)}{\eta}\left(\frac{Q^{2}}{\mu^{2}}\right)^{\eta},
$$

where $f\left(p^{2}\right)$ is a smooth test function. The subtraction term involving $f(0)$ is required only if $\eta<0$. For small $\eta$, the above definition implies the expansion

$$
\left[\frac{1}{p^{2}}\left(\frac{p^{2}}{\mu^{2}}\right)^{\eta}\right]_{*}=\frac{\delta\left(p^{2}\right)}{\eta}+\left[\frac{1}{p^{2}}\right]_{*}+\eta\left[\frac{1}{p^{2}} \ln \frac{p^{2}}{\mu^{2}}\right]_{*}+\mathcal{O}\left(\eta^{2}\right) .
$$

The singularity for $\eta \rightarrow 0$ is removed by the factor $1 / \Gamma(\eta)$ in (46). In the form given above, the expression for $J\left(p^{2}, \mu\right)$ holds as long as $\eta>-1$, which is sufficient for all practical purposes. For even smaller values of $\eta$ it would be necessary to perform further subtractions in (47) by using the double-star distributions introduced in [40]. 


\subsection{Matching conditions and anomalous dimensions}

To evaluate the resummed hard and jet functions at a common factorization scale $\mu$ requires perturbative expressions for the matching functions $C_{V}\left(Q^{2}, \mu_{h}\right)$ and $\widetilde{j}\left(L, \mu_{i}\right)$. We extract the hard coefficient at a scale $\mu_{h} \sim Q$ in the first matching step, and the associated jet function at a scale $\mu_{i} \sim Q \sqrt{1-x}$ in the second. In this way, the matching functions are free of large logarithms and can be reliably computed in fixed-order perturbation theory. We also need perturbative expressions for the anomalous dimensions $\Gamma_{\text {cusp }}, \gamma^{V}$, and $\gamma^{J}$.

The hard matching coefficient $C_{V}\left(Q^{2}, \mu\right)$ is extracted in the first matching step, when the vector current in full QCD is matched onto an effective current built out of operators in SCET. To obtain an expression for the Wilson coefficient one must compute, at a given order in $\alpha_{s}$, perturbative expressions for the photon vertex function in the two theories. The calculation is simplified greatly by performing these calculations on-shell, in which case all loop graphs in the effective theory are scaleless and hence vanish. The bare on-shell vertex function in QCD (called the on-shell quark form factor) has been studied extensively in the literature. The form factor is infrared divergent and can be regularized using dimensional regularization. The bare form factor at two-loop order was calculated long ago [41, 42, 43, 44, and recently the infrared divergent contributions have even been computed at three-loop order [45. When the (vanishing) SCET graphs are subtracted from the QCD result, the infrared poles in $1 / \epsilon$ get transformed into ultraviolet poles. To obtain the matching coefficient we introduce a renormalization factor $Z_{V}$, which absorbs these poles. We then compute

$$
C_{V}\left(Q^{2}, \mu\right)=\lim _{\epsilon \rightarrow 0} Z_{V}^{-1}\left(\epsilon, Q^{2}, \mu\right) F_{\text {bare }}\left(\epsilon, Q^{2}\right),
$$

where on the right-hand side we must also eliminate the bare coupling constant in favor of the renormalized coupling $\alpha_{s}(\mu)$. At two-loop order, we find (with $L=\ln \left(Q^{2} / \mu^{2}\right)$ and $\alpha_{s}=\alpha_{s}(\mu)$ )

$$
C_{V}\left(Q^{2}, \mu\right)=1+\frac{C_{F} \alpha_{s}}{4 \pi}\left(-L^{2}+3 L-8+\frac{\pi^{2}}{6}\right)+C_{F}\left(\frac{\alpha_{s}}{4 \pi}\right)^{2}\left[C_{F} H_{F}+C_{A} H_{A}+T_{F} n_{f} H_{f}\right],
$$

where

$$
\begin{aligned}
H_{F}= & \frac{L^{4}}{2}-3 L^{3}+\left(\frac{25}{2}-\frac{\pi^{2}}{6}\right) L^{2}+\left(-\frac{45}{2}-\frac{3 \pi^{2}}{2}+24 \zeta_{3}\right) L+\frac{255}{8}+\frac{7 \pi^{2}}{2}-\frac{83 \pi^{4}}{360}-30 \zeta_{3}, \\
H_{A}= & \frac{11}{9} L^{3}+\left(-\frac{233}{18}+\frac{\pi^{2}}{3}\right) L^{2}+\left(\frac{2545}{54}+\frac{11 \pi^{2}}{9}-26 \zeta_{3}\right) L \\
& -\frac{51157}{648}-\frac{337 \pi^{2}}{108}+\frac{11 \pi^{4}}{45}+\frac{313}{9} \zeta_{3} \\
H_{f}= & -\frac{4}{9} L^{3}+\frac{38}{9} L^{2}+\left(-\frac{418}{27}-\frac{4 \pi^{2}}{9}\right) L+\frac{4085}{162}+\frac{23 \pi^{2}}{27}+\frac{4}{9} \zeta_{3} .
\end{aligned}
$$

This result agrees with the corresponding expression given in [21]. The anomalous dimension of the vector current in SCET is obtained from the coefficient $Z_{V}^{(1)}$ of the $1 / \epsilon$ pole term via the relation

$$
\Gamma_{\text {cusp }}\left(\alpha_{s}\right) \ln \frac{Q^{2}}{\mu^{2}}+\gamma^{V}\left(\alpha_{s}\right)=2 \alpha_{s} \frac{\partial}{\partial \alpha_{s}} Z_{V}^{(1)}\left(Q^{2}, \mu\right) .
$$


Using the results of [45] the anomalous dimension can be extracted at three-loop order. We reproduce the well-known expression for the three-loop cusp anomalous dimension $\Gamma_{\text {cusp }}$ [46]. For the quantity $\gamma^{V}$, we obtain

$$
\gamma^{V}\left(\alpha_{s}\right)=-\frac{2 \alpha_{s}}{\pi}-\left(4.68-0.95 n_{f}\right)\left(\frac{\alpha_{s}}{\pi}\right)^{2}-\left(23.43-4.05 n_{f}+0.029 n_{f}^{2}\right)\left(\frac{\alpha_{s}}{\pi}\right)^{3}+\ldots
$$

The exact analytic expressions for the expansion coefficients are given in the Appendix.

The two-loop expression for the jet function has recently been obtained in [31] starting from expression (20), by which the jet function is expressed in terms of a two-point vacuum correlator in full QCD. Using those results, the two-loop matching condition for the associated jet function is found to $\mathrm{be}^{2}$

$$
\widetilde{j}(L, \mu)=1+\frac{C_{F} \alpha_{s}}{4 \pi}\left(2 L^{2}-3 L+7-\frac{2 \pi^{2}}{3}\right)+C_{F}\left(\frac{\alpha_{s}}{4 \pi}\right)^{2}\left[C_{F} J_{F}+C_{A} J_{A}+T_{F} n_{f} J_{f}\right],
$$

where

$$
\begin{aligned}
J_{F}= & 2 L^{4}-6 L^{3}+\left(\frac{37}{2}-\frac{4 \pi^{2}}{3}\right) L^{2}+\left(-\frac{45}{2}+4 \pi^{2}-24 \zeta_{3}\right) L+\frac{205}{8}-\frac{97 \pi^{2}}{12}+\frac{61 \pi^{4}}{90}-6 \zeta_{3}, \\
J_{A}= & -\frac{22}{9} L^{3}+\left(\frac{367}{18}-\frac{2 \pi^{2}}{3}\right) L^{2}+\left(-\frac{3155}{54}+\frac{11 \pi^{2}}{9}+40 \zeta_{3}\right) L \\
& +\frac{53129}{648}-\frac{155 \pi^{2}}{36}-\frac{37 \pi^{4}}{180}-18 \zeta_{3}, \\
J_{f}= & \frac{8}{9} L^{3}-\frac{58}{9} L^{2}+\left(\frac{494}{27}-\frac{4 \pi^{2}}{9}\right) L-\frac{4057}{162}+\frac{13 \pi^{2}}{9} .
\end{aligned}
$$

The anomalous dimension kernel entering (40) has been calculated at two-loop order [31. In Section 3.5 below, we will show that the difference $\left(\gamma^{J}-\gamma^{V}\right)$ multiplies the $\delta(1-z)$ term in the Altarelli-Parisi splitting function $P_{q \leftarrow q}(z)$, which has recently been calculated at threeloop order [46]. When combined with (53) this relation serves as a cross-check of the two-loop result obtained from the direct calculation in [31, and further it can be employed to extract the three-loop coefficient of the jet-function anomalous dimension. We find

$$
\gamma^{J}\left(\alpha_{s}\right)=-\frac{\alpha_{s}}{\pi}-\left(0.364-0.556 n_{f}\right)\left(\frac{\alpha_{s}}{\pi}\right)^{2}-\left(3.18-1.33 n_{f}+0.011 n_{f}^{2}\right)\left(\frac{\alpha_{s}}{\pi}\right)^{3}+\ldots
$$

The exact analytic expressions for the expansion coefficients are given in the Appendix.

\subsection{Resummation of large logarithms}

We are now ready to write down a resummed expression for the structure function $F_{2}^{\text {ns }}\left(x, Q^{2}\right)$, valid to all orders in perturbation theory and at leading power in $(1-x)$ and $\Lambda_{\mathrm{QCD}}^{2} / M_{X}^{2}$. The

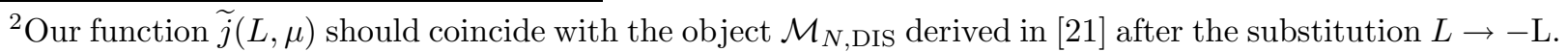
Comparing the two expressions, we disagree with the signs of the two-loop $\mathcal{O}(\mathrm{L})$ terms with color structures $C_{F} C_{A}$ and $C_{F} T_{F} n_{f}$, and with the two-loop constant terms with color structures $C_{F}^{2}$ and $C_{F} C_{A}$.
} 
result is

$$
\begin{aligned}
F_{2}^{\mathrm{ns}}\left(x, Q^{2}\right)= & \sum_{q} e_{q}^{2}\left|C_{V}\left(Q^{2}, \mu_{h}\right)\right|^{2}\left(\frac{Q^{2}}{\mu_{h}^{2}}\right)^{-2 a_{\Gamma}\left(\mu_{h}, \mu_{f}\right)} \\
& \times \exp \left[4 S\left(\mu_{h}, \mu_{f}\right)-4 S\left(\mu_{i}, \mu_{f}\right)-2 a_{\gamma^{V}}\left(\mu_{h}, \mu_{f}\right)+2 a_{\gamma^{J}}\left(\mu_{i}, \mu_{f}\right)\right] \\
& \times \tilde{j}\left(\partial_{\eta}, \mu_{i}\right) \frac{e^{-\gamma_{E} \eta}}{\Gamma(\eta)} Q^{2} \int_{x}^{1} d \xi\left[\frac{1}{Q^{2}(\xi / x-1)}\left(\frac{Q^{2}(\xi / x-1)}{\mu_{i}^{2}}\right)^{\eta}\right]_{*} \phi_{q}^{\mathrm{ns}}\left(\xi, \mu_{f}\right),
\end{aligned}
$$

where $\eta=2 a_{\Gamma}\left(\mu_{i}, \mu_{f}\right)$, as above. To leading power, we could approximate $(\xi / x-1) \rightarrow$ $(\xi-x)$, but we prefer to keep the full $x$ dependence in our numerical studies below. The "factorization scale" $\mu_{f} \equiv \mu$ is, by definition, the scale at which the parton distribution function is renormalized.

The Sudakov exponent can be simplified using the general relations

$$
\begin{aligned}
a_{\Gamma}\left(\mu_{1}, \mu_{2}\right)+a_{\Gamma}\left(\mu_{2}, \mu_{3}\right) & =a_{\Gamma}\left(\mu_{1}, \mu_{3}\right), \\
S\left(\mu_{1}, \mu_{2}\right)+S\left(\mu_{2}, \mu_{3}\right) & =S\left(\mu_{1}, \mu_{3}\right)+\ln \frac{\mu_{1}}{\mu_{2}} a_{\Gamma}\left(\mu_{2}, \mu_{3}\right) .
\end{aligned}
$$

Introducing the short-hand notations

$$
\gamma^{\phi}=\gamma^{J}-\gamma^{V}, \quad a_{\gamma^{\phi}}=a_{\gamma^{J}}-a_{\gamma^{V}},
$$

we find after a straightforward calculation

$$
\begin{aligned}
F_{2}^{\mathrm{ns}}\left(x, Q^{2}\right)= & \sum_{q} e_{q}^{2}\left|C_{V}\left(Q^{2}, \mu_{h}\right)\right|^{2}\left(\frac{Q^{2}}{\mu_{h}^{2}}\right)^{-2 a_{\Gamma}\left(\mu_{h}, \mu_{i}\right)} \exp \left[4 S\left(\mu_{h}, \mu_{i}\right)-2 a_{\gamma^{V}}\left(\mu_{h}, \mu_{i}\right)\right] \\
& \times \exp \left[2 a_{\gamma^{\phi}}\left(\mu_{i}, \mu_{f}\right)\right] \tilde{j}\left(\ln \frac{Q^{2}}{\mu_{i}^{2}}+\partial_{\eta}, \mu_{i}\right) \frac{e^{-\gamma_{E} \eta}}{\Gamma(\eta)} \int_{x}^{1} d \xi \frac{\phi_{q}^{\mathrm{ns}}\left(\xi, \mu_{f}\right)}{\left[(\xi / x-1)^{1-\eta}\right]_{*}} .
\end{aligned}
$$

The remaining integral can be performed by noting that, on very general grounds, the behavior of the parton distribution function near the endpoint can be parameterized as

$$
\left.\phi_{q}^{\mathrm{ns}}\left(\xi, \mu_{f}\right)\right|_{\xi \rightarrow 1}=\mathcal{N}\left(\mu_{f}\right)(1-\xi)^{b\left(\mu_{f}\right)}[1+\mathcal{O}(1-\xi)]
$$

where $b\left(\mu_{f}\right)>0$. We will see in the next subsection that this functional form is preserved under RG evolution. Defining a $K$ factor by the ratio

$$
K\left(x, Q^{2}, \mu_{f}\right)=\frac{F_{2}^{\mathrm{ns}}\left(x, Q^{2}\right)}{\sum_{q} e_{q}^{2} x \phi_{q}^{\mathrm{ns}}\left(x, \mu_{f}\right)},
$$

we now obtain our final expression

$$
\begin{aligned}
K\left(x, Q^{2}, \mu_{f}\right) & =\left|C_{V}\left(Q^{2}, \mu_{h}\right)\right|^{2}\left(\frac{Q^{2}}{\mu_{h}^{2}}\right)^{-2 a_{\Gamma}\left(\mu_{h}, \mu_{i}\right)} \exp \left[4 S\left(\mu_{h}, \mu_{i}\right)-2 a_{\gamma^{V}}\left(\mu_{h}, \mu_{i}\right)\right] \\
& \times \exp \left[2 a_{\gamma^{\phi}}\left(\mu_{i}, \mu_{f}\right)\right]\left(\frac{1-x}{x}\right)^{\eta} \tilde{j}\left(\ln \frac{Q^{2}}{\mu_{i}^{2}} \frac{1-x}{x}+\partial_{\eta}, \mu_{i}\right) \frac{e^{-\gamma_{E} \eta} \Gamma\left(1+b\left(\mu_{f}\right)\right)}{\Gamma\left(1+b\left(\mu_{f}\right)+\eta\right)},
\end{aligned}
$$


Table 1: Different approximation schemes for the evaluation of the resummed factorization formula (63)

\begin{tabular}{ccll|ccc}
\hline \hline RG-impr. PT & Log. Approx. & \multicolumn{2}{c|}{ Accuracy $\sim \alpha_{s}^{n} L^{k}$} & $\Gamma_{\text {cusp }}$ & $\gamma^{V}, \gamma^{J}$ & $C_{V}, \tilde{j}$ \\
\hline- & LL & $n+1 \leq k \leq 2 n\left(\alpha_{s}^{-1}\right)$ & 1-loop & tree-level & tree-level \\
LO & NLL & $n \leq k \leq 2 n$ & $\left(\alpha_{s}^{0}\right)$ & 2-loop & 1-loop & tree-level \\
NLO & NNLL & $n-1 \leq k \leq 2 n$ & $\left(\alpha_{s}\right)$ & 3-loop & 2-loop & 1-loop \\
NNLO & NNNLL & $n-2 \leq k \leq 2 n$ & $\left(\alpha_{s}^{2}\right)$ & 4-loop & 3-loop & 2-loop \\
\hline \hline
\end{tabular}

where as before $\eta=2 a_{\Gamma}\left(\mu_{i}, \mu_{f}\right)$. In this expression, the dependence on the two physical scales $Q^{2}$ and $M_{X}^{2}=Q^{2} \frac{1-x}{x}$ (neglecting the nucleon mass) is completely explicit. Our exact result is independent of the scales $\mu_{h}$ and $\mu_{i}$, at which QCD is matched onto the intermediate and final effective theories, $\operatorname{SCET}(h c, \bar{c}, s c)$ and $\operatorname{SCET}(\bar{c}, s c)$, respectively. In practice, a residual scale dependence remains once we truncate the perturbative expansions of the various objects in the factorization formula. The final answer simplifies further if we choose the "natural" values of the two matching scales, $\mu_{h}=Q$ and $\mu_{i}=M_{X}$. However, we prefer to vary the matching scales over some reasonable range and take the variation of the results as an estimate of higher-order perturbative effects. Note that by definition the $K$ factor does depend on the choice of the factorization scale $\mu_{f}$. It is conventional in the literature on DIS to identify $\mu_{f}$ with the hard scale $Q$. However, from the point of view of an effective field theory it would be more natural to choose $\mu_{f}$ below the intermediate matching scale $\mu_{i} \sim Q \sqrt{1-x}$. A typical choice would be $\mu_{f}$ of order a few $\mathrm{GeV}$, independent of the dynamical variables $x$ and $Q$.

In (60) and (63) we have accomplished the complete resummation of threshold logarithms for $F_{2}$ directly in momentum space. That the final answer is a convolution (rather than a product) of a hard-scattering kernel with the parton distribution function is reflected in the non-trivial dependence on the hadronic parameter $b\left(\mu_{f}\right)$ describing the large- $\xi$ behavior of $\phi_{q}^{\text {ns }}\left(\xi, \mu_{f}\right)$. Our factorized expression for the DIS structure function is very similar to that for the $B \rightarrow X_{s} \gamma$ decay rate derived in [25]. Although the hard functions and soft matrix elements differ, the jet function is the same in both cases. An important advantage of our momentum-space approach is that in the limit where the two matching scales are set equal to the factorization scale, $\mu_{h}=\mu_{i}=\mu_{f}$, the resummed results (60) and (63) automatically reduce to the corresponding expressions valid in fixed-order perturbation theory (expanded about $x=1$ ). Consequently, it is straightforward to match our resummed expressions onto fixed-order calculations valid outside the threshold region.

The right-hand sides of (60) and (63) can be evaluated at any desired order in resummed perturbation theory. Table 1 shows what is required to obtain different levels of accuracy in the perturbative evaluation of the result. In this work we adopt the counting scheme of RGimproved perturbation theory, where at leading-order (LO) one includes all terms of order 1 , at next-to-leading order (NLO) one includes all terms of order $\alpha_{s}$, and at next-to-next-toleading order (NNLO) one includes all terms of order $\alpha_{s}^{2}$. We count the large logarithms 

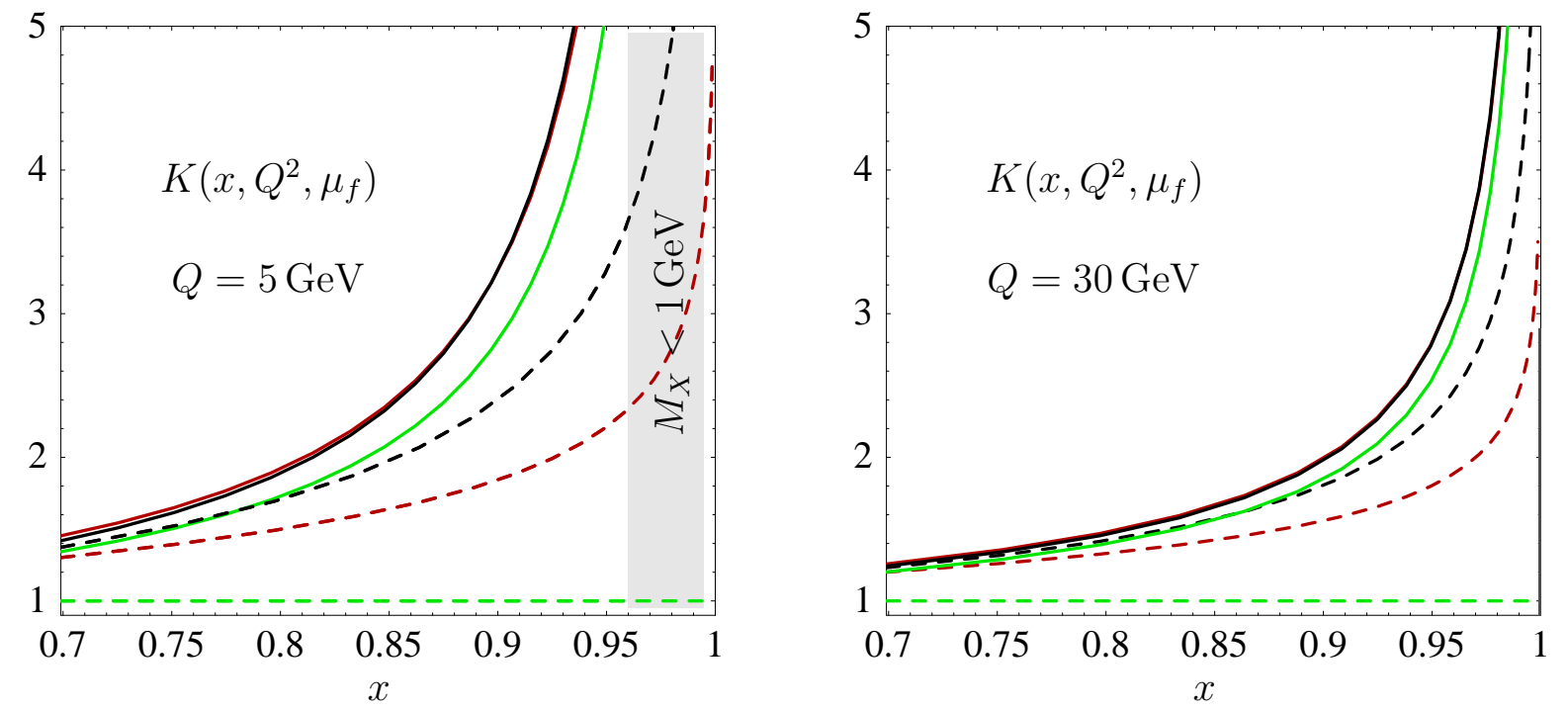

Figure 5: Comparison between fixed-order (dashed) and resummed results (solid) for the $K$ factor. The green curves are the LO result, red NLO, black NNLO. For the resummed result, we set $\mu_{h}=Q, \mu_{i}=M_{X}, \mu_{f}=Q$, and $b\left(\mu_{f}\right)=4$. The fixed-order result is obtained by setting all scales equal to $\mu_{f}$.

$L \in\left\{\ln \mu_{h} / \mu_{i}, \ln \mu_{i} / \mu_{f}, \ln (1-x)\right\}$ like $1 / \alpha_{s}$. In the literature on DIS, the LO approximation is also referred to as the next-to-leading logarithmic (NLL) approximation, the NLO result is referred to as the next-to-next-to-leading logarithmic (NNLL) approximation, etc. The leading logarithmic (LL) approximation is listed only for completeness, as it neglects terms that are parametrically much larger than 1.

In Figure 5. we compare the fixed-order calculation of the $K$ factor with the resummed result for $Q=5 \mathrm{GeV}$ and $Q=30 \mathrm{GeV}$. For the resummed result we use the default choice of scales $\mu_{h}=Q, \mu_{i}=M_{X}=Q \sqrt{\frac{1-x}{x}}$ and take the asymptotic form of the parton distribution (61) with $b\left(\mu_{f}\right)=4$ in both cases. Following common practice we choose $\mu_{f}=Q$ for the factorization scale. In this case the quantity $\eta<0$, and because of the factor $\left(\frac{1-x}{x}\right)^{\eta}$ in (63) the resummed results diverge as $x$ approaches 1 . The figure illustrates that higher-order corrections become important as $x \rightarrow 1$, and that fixed-order perturbation theory is no longer adequate in this limit. The magnitude of the $K$ factor can be reduced by adopting a lower choice for the factorization scale, which is more in line with the philosophy of an effective field-theory approach. For example, we may consider taking $\mu_{f} \approx M_{X}(x=0.9) \approx Q / 3$, corresponding to a typical hadronic invariant mass in the endpoint region. The corresponding results are shown in Figure 6. We observe that with such a choice of the factorization scale the $K$ factor takes more moderate values, and also that the effects of resummation are less significant.

In Figure [7. we show the scale dependence of the result obtained by varying the hard and intermediate scales by a factor of 2 about their default values. The figure shows a dramatic reduction in scale uncertainty when going from LO to NNLO. It also suggests that varying the two matching scales individually by a factor of 2 may overestimate the perturbative un- 

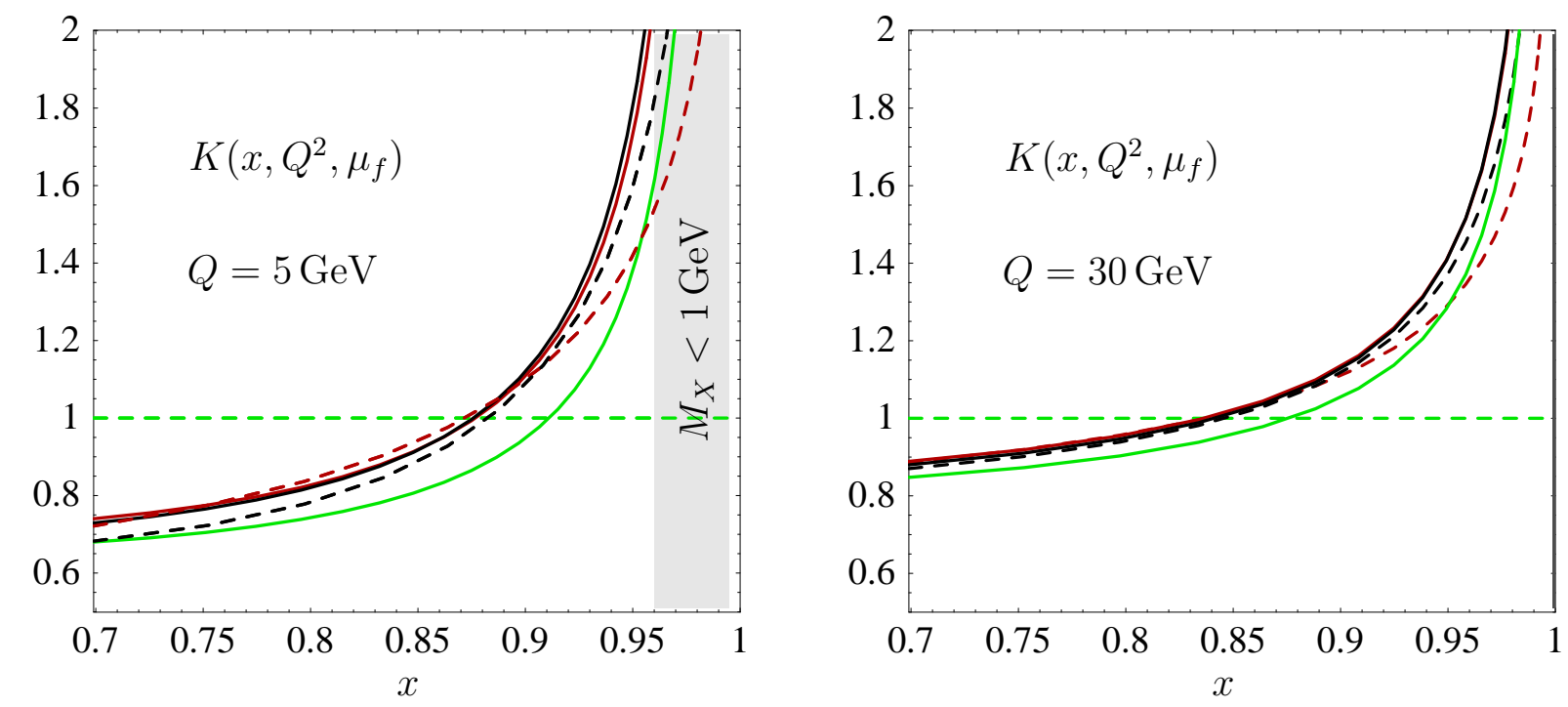

Figure 6: Same as Figure 5 but with a lower choice of the factorization scale. Specifically, we take $\mu_{f}=1.5 \mathrm{GeV}$ for $Q=5 \mathrm{GeV}$ (left), and $\mu_{f}=10 \mathrm{GeV}$ for $Q=30 \mathrm{GeV}$ (right).
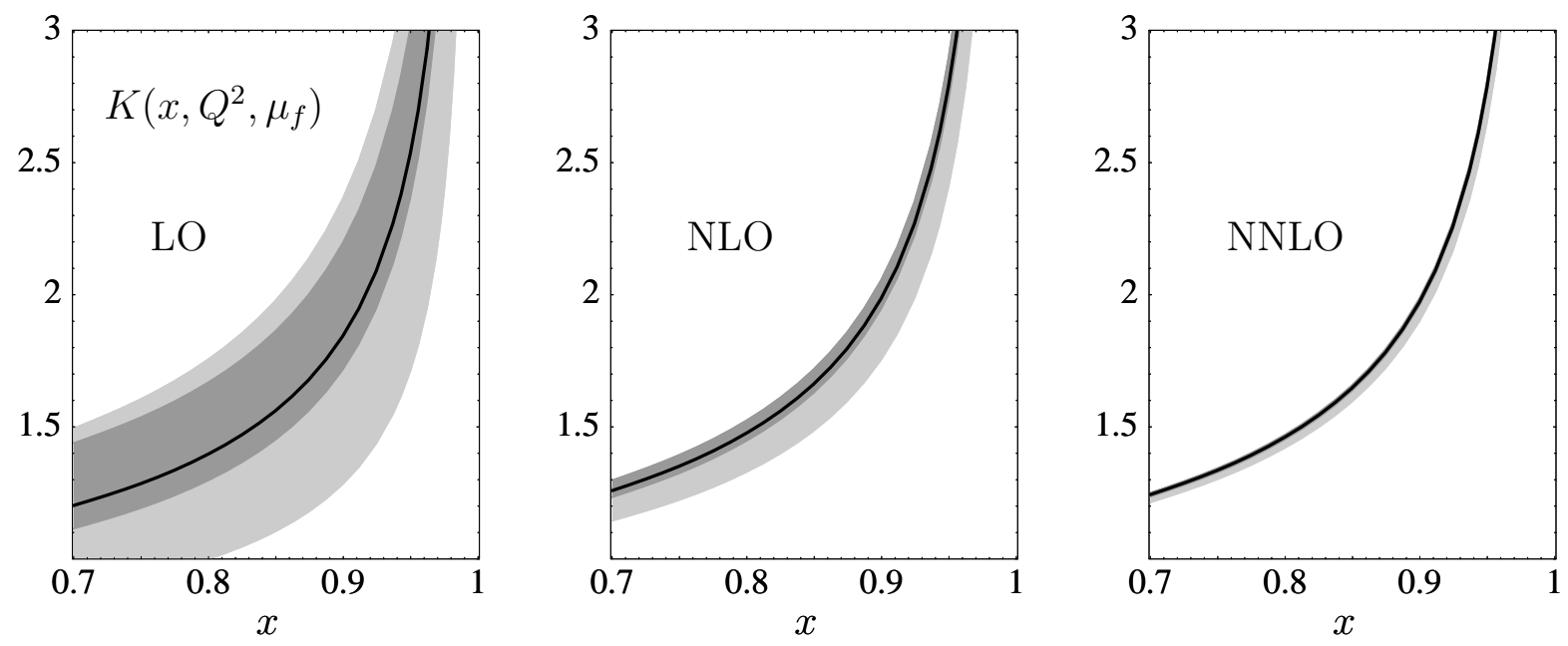

Figure 7: Scale variation of the $K$ factor at $Q=30 \mathrm{GeV}$. The light-gray band is obtained by varying $M_{X} / 2<\mu_{i}<2 M_{X}$, while the dark-gray band arises from varying the hard scale $Q / 2<\mu_{h}<2 Q$. We set $\mu_{f}=30 \mathrm{GeV}$ and $b\left(\mu_{f}\right)=4$. 

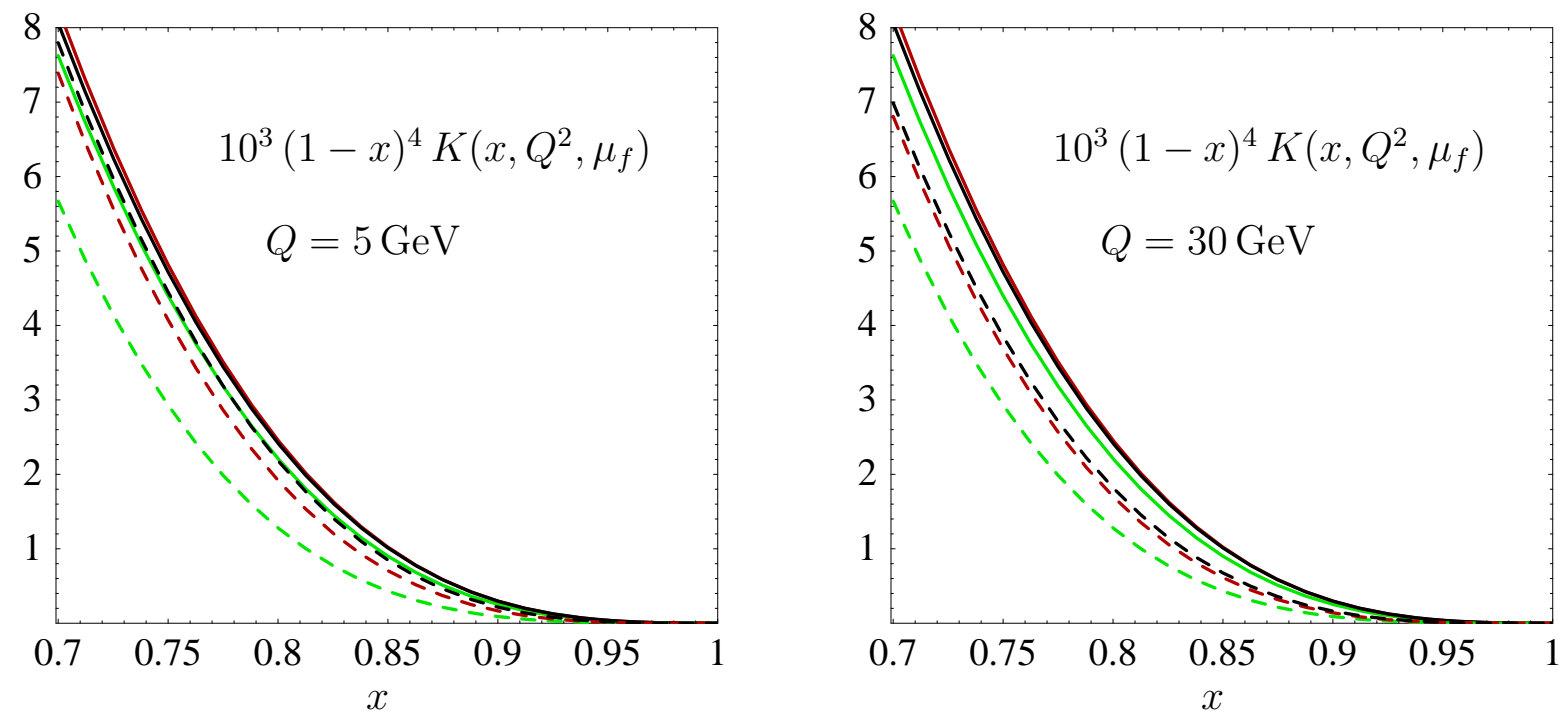

Figure 8: Same as Figure 5, but now including the suppression factor $(1-x)^{b}$ with $b\left(\mu_{f}\right)=4$ from the parton distribution function.

certainty, because the higher-order results lie near the center of the large band obtained by varying the renormalization scales in the low-order ones. A variation of the scales by a factor of $\sqrt{2}$ better represents the uncertainty in the present case. Furthermore, it seems reasonable to perform the scale variations in such a way that the hierarchy of scales $\mu_{h}>\mu_{i}$ is preserved.

We stress that the resummation of large logarithms accomplished in (63) is under perturbative control as long as $(1-x) \gg \Lambda_{\mathrm{QCD}}^{2} / Q^{2}$, since only then is the intermediate matching scale $\mu_{i} \sim Q \sqrt{1-x} \gg \Lambda_{\mathrm{QCD}}$ a short-distance scale. Physically, this condition is equivalent to saying that the final-state jet can be treated in an inclusive way using a partonic language. Numerically, we can assume that perturbation theory at the jet scale breaks down in the region where $M_{X}<1 \mathrm{GeV}$. We illustrate this boundary in our $x$-space results with the gray band in Figure 5. For $Q=5 \mathrm{GeV}$, our approach is valid as long as $x<0.96$. For $Q=30 \mathrm{GeV}$, it extends all the way to $x<0.999$, so that in this case the band is not visible on the scale of the plot. While our theoretical description breaks down very close to the endpoint, we note that weighted integrals of the jet function over an interval $x \in\left[x_{0}, 1\right]$ can be calculated starting from (60) as long as $x_{0}$ is in the short-distance domain.

Experimentally, structure functions at large $x$ are very difficult to measure, because of the rapid decrease of the parton distribution function as $x \rightarrow 1$. This is illustrated in Figure 8 , where we include the suppression factor from the parton distribution. Because of this strong suppression, there are no measurements of the non-singlet structure function for $x>0.9$ 47, 48, 49]. The experiments that probed the highest $x$-values were fixed-target experiments in the 1970s and 1980s at SLAC [50] and the BCDMS experiment at CERN [51]. Newer experiments only cover $x \leq 0.65$. As a consequence, the threshold resummation in DIS is currently of limited phenomenological importance. However, the resummation is relevant for $W$ or Higgs production at hadron colliders, which can also be analyzed with the methods developed here. 


\subsection{Parton evolution near the endpoint}

The easiest way to derive the evolution equation for the parton distribution function in the limit $x \rightarrow 1$ is to use that the factorized expression (26) for the structure function $F_{2}$ must be independent of the arbitrary renormalization scale $\mu$, and to combine this information with the known scale dependences of the hard and jet functions, given in (36) and (40). This yields

$$
\begin{aligned}
\frac{d}{d \ln \mu} \phi_{q}^{\mathrm{ns}}(\xi, \mu) & =2 \gamma^{\phi}\left(\alpha_{s}\right) \phi_{q}^{\mathrm{ns}}(\xi, \mu)+2 \Gamma_{\text {cusp }}\left(\alpha_{s}\right) \int_{\xi}^{1} d \xi^{\prime} \frac{\phi_{q}^{\mathrm{ns}}\left(\xi^{\prime}, \mu\right)}{\left[\xi^{\prime}-\xi\right]_{*}} \\
& =\int_{\xi}^{1} \frac{d z}{z} P_{q \leftarrow q}^{(\text {endpt })}(z) \phi_{q}^{\mathrm{ns}}\left(\frac{\xi}{z}, \mu\right),
\end{aligned}
$$

where

$$
P_{q \leftarrow q}^{\text {(endpt) }}(z)=\frac{2 \Gamma_{\text {cusp }}\left(\alpha_{s}\right)}{(1-z)_{+}}+2 \gamma^{\phi}\left(\alpha_{s}\right) \delta(1-z)
$$

is the $z \rightarrow 1$ limit of the Altarelli-Parisi splitting function $P_{q \leftarrow q}(z)$, which is known from direct calculation at three-loop order [46]. The asymptotic form of the splitting function near the endpoint given above holds to all orders in perturbation theory, up to corrections of order $(1-z)$. Recall that the anomalous dimension $\gamma^{\phi}$ was defined as the difference of the anomalous dimensions $\gamma^{J}$ and $\gamma^{V}$ of the jet function and SCET current, see (59). Relation (65) thus provides a check of our two-loop results for these anomalous dimensions, and it furthermore allows us to deduce the value of the three-loop coefficient $\gamma_{2}^{J}$ given in relation (97) of the Appendix.

The exact solution to the evolution equation (64) can be found in analogy with (45). It reads

$$
\phi_{q}^{\mathrm{ns}}\left(\xi, \mu_{f}\right)=\exp \left[2 a_{\gamma^{\phi}}\left(\mu_{f}, \mu_{0}\right)\right] \frac{e^{-\gamma_{E} \sigma}}{\Gamma(\sigma)} \int_{\xi}^{1} d \xi^{\prime} \frac{\phi_{q}^{\mathrm{ns}}\left(\xi^{\prime}, \mu_{0}\right)}{\left(\xi^{\prime}-\xi\right)^{1-\sigma}},
$$

where this time $\sigma=2 a_{\Gamma}\left(\mu_{f}, \mu_{0}\right)$, and $\mu_{0}$ denotes the scale at which the initial condition for

$\phi_{q}^{\text {ns }}$ is given. For the hadronic parameters $\mathcal{N}$ and $b$ governing the asymptotic behavior of the parton distribution function in (61), this relation implies

$$
\begin{aligned}
b\left(\mu_{f}\right) & =b\left(\mu_{0}\right)+2 a_{\Gamma}\left(\mu_{f}, \mu_{0}\right), \\
\mathcal{N}\left(\mu_{f}\right) & =\mathcal{N}\left(\mu_{0}\right) \exp \left[2 a_{\gamma^{\phi}}\left(\mu_{f}, \mu_{0}\right)\right] \frac{e^{\gamma_{E} b\left(\mu_{0}\right)} \Gamma\left(1+b\left(\mu_{0}\right)\right)}{e^{\gamma_{E} b\left(\mu_{f}\right)} \Gamma\left(1+b\left(\mu_{f}\right)\right)} .
\end{aligned}
$$

These evolution equations ensure that the $\mu_{f}$ dependence on the two sides of relation (63) is indeed the same. The first result is particularly simple and interesting. Since $a_{\Gamma}\left(\mu_{f}, \mu_{0}\right)>0$ for $\mu_{f}>\mu_{0}$, it follows that the coefficient $b(\mu)$ increases with $\mu$, a fact incompatible with the naive counting rule result $b=3[52,53]$. In other words, such a counting rule could possibly hold only at a specific renormalization point.

\section{Connection with the standard approach}

The conventional approach to threshold resummation in DIS proceeds via moment space and inverse Mellin transformations [1, 2]. The purpose of this section is twofold; first, to show that 
our momentum-space resummation is formally equivalent to the conventional resummation order by order in perturbation theory, and second, to point out and quantify the theoretical and numerical differences that appear in applications to physical quantities such as the DIS structure function.

We first recall some details of the conventional approach to threshold resummation in moment space. By taking moments of the structure function $F_{2}^{\text {ns }}$, convolution integrals such as (11) or (26) can be brought into product form. The traditional way of writing the result is

$$
F_{2, N}^{\mathrm{ns}}\left(Q^{2}\right)=\int_{0}^{1} d x x^{N-1} F_{2}^{\mathrm{ns}}\left(x, Q^{2}\right)=C_{N}\left(Q^{2}, \mu_{f}\right) \sum_{q} e_{q}^{2} \phi_{q, N+1}^{\mathrm{ns}}\left(\mu_{f}\right) .
$$

where the moments of $C\left(Q^{2}, z, \mu\right)$ and $\phi_{q}^{\mathrm{ns}}(\xi, \mu)$ are defined in analogy to those of $F_{2}^{\mathrm{ns}}\left(x, Q^{2}\right)$. For large $N$, the function $C_{N}$ is then split into two pieces according to

$$
C_{N}\left(Q^{2}, \mu_{f}\right)=g_{0}\left(Q^{2}, \mu_{f}\right) \exp \left[G_{N}\left(Q^{2}, \mu_{f}\right)\right]+\mathcal{O}\left(\frac{1}{N}\right)
$$

where $g_{0}$ contains all $N$-independent contributions, while the function $G_{N}$ contains logarithms of the form $\ln ^{k} N$. The limit $x \rightarrow 1$ in momentum space corresponds to the limit $N \rightarrow \infty$ in moment space, so this formula achieves the exponentiation of large threshold logarithms. The resummation exponent $G_{N}$ is written as

$$
G_{N}\left(Q^{2}, \mu_{f}\right)=\int_{0}^{1} d z \frac{z^{N-1}-1}{1-z}\left[\int_{\mu_{f}^{2}}^{(1-z) Q^{2}} \frac{d k^{2}}{k^{2}} A_{q}\left(\alpha_{s}(k)\right)+B_{q}\left(\alpha_{s}(Q \sqrt{1-z})\right)\right],
$$

where the functions $A_{q}$ and $B_{q}$ are universal radiation factors determined by matching with results from fixed-order perturbation theory.

We shall now derive an equation relating the objects $g_{0}, A_{q}$, and $B_{q}$ in (69) and (70) to the matching coefficients and anomalous dimensions defined in effective field theory. We begin by transforming the factorization formula (26) into Mellin space, obtaining the product form

$$
F_{2, N}^{\mathrm{ns}}\left(Q^{2}\right)=\left|C_{V}\left(Q^{2}, \mu_{f}\right)\right|^{2} J_{N}\left(Q^{2}, \mu_{f}\right) \sum_{q} e_{q}^{2} \phi_{q, N+1}^{\mathrm{ns}}\left(\mu_{f}\right)
$$

which is valid up to corrections in $1 / N$. The Mellin-transformed jet function is defined as

$$
J_{N}\left(Q^{2}, \mu\right)=\int_{0}^{Q^{2}} d p^{2}\left(1-\frac{p^{2}}{Q^{2}}\right)^{N-1} J\left(p^{2}, \mu\right) .
$$

It was shown in 31] that for large $N$ the jet-function moments $J_{N}$ are given by

$$
J_{N}\left(Q^{2}, \mu\right)=\widetilde{j}\left(\ln \frac{Q^{2}}{\bar{N} \mu^{2}}, \mu\right)+\mathcal{O}\left(\frac{1}{N}\right), \quad \bar{N} \equiv e^{\gamma_{E}} N
$$

and hence obey the same evolution equation (43) as the associated jet function. Using this connection along with the results derived in Section 3, the resummed coefficient function $C_{N}$ 
in (68) can be written as

$$
\begin{aligned}
C_{N}\left(Q^{2}, \mu_{f}\right)= & \left|C_{V}\left(Q^{2}, \mu_{h}\right)\right|^{2}\left(\frac{Q^{2}}{\mu_{h}^{2}}\right)^{-2 a_{\Gamma}\left(\mu_{h}, \mu_{i}\right)} \exp \left[4 S\left(\mu_{h}, \mu_{i}\right)-2 a_{\gamma^{V}}\left(\mu_{h}, \mu_{i}\right)\right] \\
& \times \exp \left[2 a_{\gamma^{\phi}}\left(\mu_{i}, \mu_{f}\right)-2 \ln \bar{N} a_{\Gamma}\left(\mu_{i}, \mu_{f}\right)\right] \tilde{j}\left(\ln \frac{Q^{2}}{\bar{N} \mu_{i}^{2}}, \mu_{i}\right)+\mathcal{O}\left(\frac{1}{N}\right) .
\end{aligned}
$$

We now adopt the "natural" scale choices $\mu_{h}=Q$ and $\mu_{i}=Q / \sqrt{\bar{N}}$, which are implicit in most treatments of threshold resummation in the literature. This allows us to compare with the standard expression (69), but as we will discuss at the end of this section, this scale choice becomes problematic when the expressions for the moments are transformed back to $x$-space. Next, we express the RG functions $S\left(\mu_{1}, \mu_{2}\right)$ and $a_{n}\left(\mu_{1}, \mu_{2}\right)$ defined in (38) in terms of integrals over the appropriate anomalous dimensions. After a straightforward calculation, this leads to

$$
\begin{aligned}
g_{0}^{\mathrm{SCET}}\left(Q^{2}, \mu_{f}\right)= & \left|C_{V}\left(Q^{2}, Q\right)\right|^{2} \widetilde{j}(0, Q) \exp \left[\int_{\mu_{f}^{2}}^{Q^{2}} \frac{d k^{2}}{k^{2}} \gamma^{\phi}\left(\alpha_{s}(k)\right)\right] \\
G_{N}^{\mathrm{SCET}}\left(Q^{2}, \mu_{f}\right)= & \int_{Q^{2} / \bar{N}}^{Q^{2}} \frac{d k^{2}}{k^{2}}\left[\ln \frac{k^{2}}{Q^{2}} \Gamma_{\text {cusp }}\left(\alpha_{s}(k)\right)-\gamma^{J}\left(\alpha_{s}(k)\right)-\frac{d \ln \widetilde{j}(0, k)}{d \ln k^{2}}\right] \\
& -\ln \bar{N} \int_{\mu_{f}^{2}}^{Q^{2} / \bar{N}} \frac{d k^{2}}{k^{2}} \Gamma_{\text {cusp }}\left(\alpha_{s}(k)\right)
\end{aligned}
$$

where we have defined the split between the two terms such that the expression for $G_{N}$ obtained in the large- $N$ limit vanishes for $\bar{N} \rightarrow 1$.

Our next task is to bring the exponent $G_{N}$ from the standard result (70) into a form resembling the SCET result (75). Since the running coupling $\alpha_{s}(k)$ depends on its argument logarithmically, a helpful identity is (for integer $k \geq 0$ )

$$
\int_{0}^{1} d z \frac{z^{N-1}-1}{1-z} \ln ^{k}(1-z)=\frac{1}{k+1} I_{k+1}\left(\ln \frac{1}{\bar{N}}\right)+\mathcal{O}\left(\frac{1}{N}\right)
$$

where

$$
I_{n}(x)=\partial_{\epsilon}^{n}\left[e^{\epsilon\left(x+\gamma_{E}\right)} \Gamma(1+\epsilon)\right]_{\epsilon \rightarrow 0}
$$

are $n$-th order polynomials defined in [25]. With the help of these relations we find that for large $N$

$$
\begin{aligned}
G_{N}\left(Q^{2}, \mu_{f}\right)= & \int_{Q^{2} / \bar{N}}^{Q^{2}} \frac{d k^{2}}{k^{2}}\left[\ln \frac{k^{2}}{Q^{2}} A_{q}\left(\alpha_{s}(k)\right)-B_{q}\left(\alpha_{s}(k)\right)\right]+\Delta G\left(\frac{Q}{\sqrt{\bar{N}}}\right) \\
& -\ln \bar{N} \int_{\mu_{f}^{2}}^{Q^{2} / \bar{N}} \frac{d k^{2}}{k^{2}} A_{q}\left(\alpha_{s}(k)\right)+\mathcal{O}\left(\frac{1}{N}\right),
\end{aligned}
$$

where

$$
\Delta G(\mu)=\sum_{k=1}^{\infty} \frac{I_{k+1}(0)}{(k+1) !}\left[A_{q}^{(k-1)}\left(\alpha_{s}(\mu)\right)+B_{q}^{(k)}\left(\alpha_{s}(\mu)\right)\right]
$$


and $A_{q}^{(n)}, B_{q}^{(n)}$ denote the $n$-th derivatives of $A_{q}$ and $B_{q}$ with respect to $\ln \mu^{2}$. The perturbative expansion of $\Delta G$ starts at order $\alpha_{s}$. Contrary to the SCET expression in (75), the result (78) does not vanish for $\bar{N} \rightarrow 1$. The overall normalization of $G_{N}\left(Q^{2}, \mu_{f}\right)$ is a matter of convention, since it can be absorbed into $g_{0}\left(Q^{2}, \mu_{f}\right)$. Taking the difference in normalization into account, the two definitions underlying (75) and (78) are connected by

$$
\begin{aligned}
g_{0}\left(Q^{2}, \mu_{f}\right) & =g_{0}^{\mathrm{SCET}}\left(Q^{2}, \mu_{f}\right) \exp [-\Delta G(Q)], \\
G_{N}\left(Q^{2}, \mu_{f}\right) & =G_{N}^{\mathrm{SCET}}\left(Q^{2}, \mu_{f}\right)+\Delta G(Q) .
\end{aligned}
$$

At the expense of a proliferation of $\gamma_{E}$ terms in the perturbative expressions, one can equally well normalize $G_{1}\left(Q^{2}, \mu_{f}\right)=0$. This normalization condition is adopted, e.g., in [4].

Equation (78) is consistent with (75) if we identify $A_{q}\left(\alpha_{s}\right)=\Gamma_{\text {cusp }}\left(\alpha_{s}\right)$ with the cusp anomalous dimension, and furthermore require that

$$
B_{q}\left(\alpha_{s}(\mu)\right)+\frac{d \Delta G(\mu)}{d \ln \mu^{2}}=\gamma^{J}\left(\alpha_{s}(\mu)\right)+\frac{d \ln \widetilde{j}(0, \mu)}{d \ln \mu^{2}} .
$$

This formula can be rearranged to read

$$
e^{\gamma_{E} \nabla} \Gamma(1+\nabla) B_{q}\left(\alpha_{s}\right)=\gamma^{J}\left(\alpha_{s}\right)+\nabla \ln \tilde{j}(0, \mu)-\left[e^{\gamma_{E} \nabla} \Gamma(\nabla)-\frac{1}{\nabla}\right] \Gamma_{\text {cusp }}\left(\alpha_{s}\right),
$$

where

$$
\nabla=\frac{d}{d \ln \mu^{2}}=\frac{\beta\left(\alpha_{s}\right)}{2} \frac{\partial}{\partial \alpha_{s}},
$$

and the differential operators are defined by their Taylor expansions. Evaluating (82) in perturbation theory we obtain ${ }^{3}$

$$
\begin{aligned}
& B_{q, 1}=\gamma_{0}^{J} \\
& B_{q, 2}=\gamma_{1}^{J}-\beta_{0} b_{0}^{(1)} \\
& B_{q, 3}=\gamma_{2}^{J}-\beta_{1} b_{0}^{(1)}-\beta_{0}\left[2 b_{0}^{(2)}-\left(b_{0}^{(1)}\right)^{2}+\frac{\pi^{2}}{6}\left(\gamma_{0}^{J}\right)^{2}-2 \zeta_{3} \Gamma_{0} \gamma_{0}^{J}+\frac{\pi^{4}}{360} \Gamma_{0}^{2}\right],
\end{aligned}
$$

where the one- and two-loop matching coefficients $b_{0}^{(1)}$ and $b_{0}^{(2)}$ have been calculated in 31. Computing the first three $B_{q, n}$ coefficients using the three-loop result for the anomalous dimension $\gamma^{J}$ given in the Appendix, we find agreement with the expressions derived in [4]. Ref. [17] identified the function $B_{q}$ with the jet-function anomalous dimension $\gamma^{J}$, which is incorrect already at two-loop order.

Equations (82) and (84) provide the desired relations between the function $B_{q}$ of the standard approach and the field-theoretical objects defined in the effective theory. Obviously, the connection between the various objects is highly non-trivial. This explains, perhaps, why it has proven difficult in the past to translate between the standard formalism and the approach

\footnotetext{
${ }^{3}$ A relation similar to (82) has been derived in [21]; however, there is a typo in the last equation in (75) of that paper, which is the analog of our relation between $B_{q, 3}$ and $\gamma_{2}^{J}$.
} 
based on SCET. The deeper reason is that in the conventional approach the RG evolution equations of SCET are replaced by a different set of partial differential equations [1, 2], whose solution is equivalent to our solution but not structurally identical to it. In particular, there are theoretical and numerical differences between the moment-space and momentum-space resummation procedures. Some of these are explicit in the particular form of the resummation exponent (70) obtained in moment space, and some become apparent only when performing the inverse Mellin transform. We conclude this section by examining these differences in more detail.

A troublesome feature of the conventional moment-space approach is that the integrals over the coupling constant in the resummation exponent run over the region where $\alpha_{s}(\mu)$ is evaluated at very small values of $\mu$. To leading order, the coupling behaves as

$$
\alpha_{s}(\mu)=\frac{4 \pi}{\beta_{0} \ln \left(\mu^{2} / \Lambda_{\mathrm{QCD}}^{2}\right)}
$$

and becomes infinite at the scale $\mu=\Lambda_{\mathrm{QCD}}$. When the integration variable $z$ in (70) approaches 1, the resummation exponent becomes sensitive to this Landau-pole singularity in the running coupling. As a result the integral is ambiguous, since one can arbitrarily choose a prescription for dealing with the pole. We can estimate the magnitude of the ambiguity by taking the difference of the $z$-integral evaluated above or below the Landau pole in (70). The result is

$$
\Delta G_{N}=-\frac{2 \pi i}{\beta_{0}}\left(\Gamma_{0}+\gamma_{0}^{J}\right)(N-1) \frac{\Lambda_{\mathrm{QCD}}^{2}}{Q^{2}}+\mathcal{O}\left(\frac{N^{2} \Lambda_{\mathrm{QCD}}^{4}}{Q^{4}}\right),
$$

which is of the form of a power correction of order $\Lambda_{\mathrm{QCD}}^{2} / M_{X}^{2}$. Note that this ambiguity never appears in the momentum-space formulation, and should therefore be interpreted as an artifact of resummation in moment space. As stressed earlier, the Landau-pole ambiguity does not imply an infrared renormalon-pole ambiguity of the same strength $\Lambda_{\mathrm{QCD}}^{2} / M_{X}^{2}$. To show that $G_{N}$ is indeed affected by a corresponding renormalon pole, one needs to evaluate the exponent in the large- $\beta_{0}$ limit, a fixed-order truncation of this quantity is not sufficient [7. On general grounds, one expects anomalous dimensions to be free of infrared renormalons, so that the renormalon poles enter only through the associated jet function $\tilde{j}(0, \mu)$ in (82). In the effective theory, renormalons affect only the matching coefficients, $C_{V}$ and $\widetilde{j}$, and will always be commensurate with power-suppressed operators. RG evolution, on the other hand, is driven by anomalous dimensions which are free of renormalons.

Further differences become apparent when studying the inverse Mellin transformation needed to obtain the physical momentum-space results from the moment-space expressions. While our result (63) obtained directly in $x$-space is completely analytical, the inverse Mellin transform can only be performed numerically, by evaluating the integral

$$
F_{2}^{\mathrm{ns}}\left(x, Q^{2}\right)=\frac{1}{2 \pi i} \int_{c-i \infty}^{c+i \infty} d N x^{-N} C_{N}\left(Q^{2}, \mu_{f}\right) \phi_{q, N+1}^{\mathrm{ns}}\left(\mu_{f}\right) .
$$

The Mellin inversion is actually ambiguous, because the expression for $C_{N}$ has a Landau pole for large $N$. We deal with this pole by adopting the so-called minimal prescription [5], which amounts to excluding the Landau pole from the integration contour by choosing the constant 

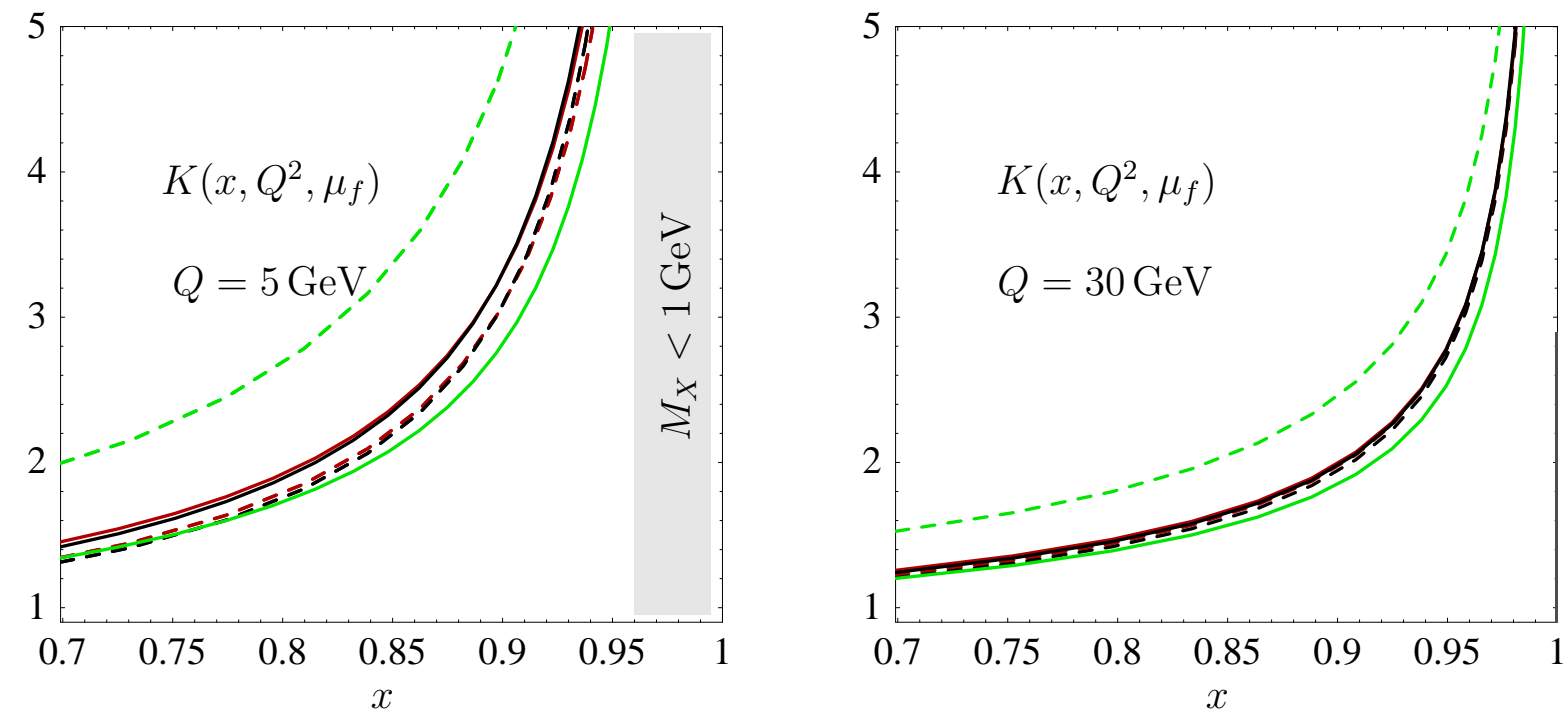

Figure 9: Comparison between Mellin-inverted moment space results (dashed) and results obtained in directly in $x$-space (solid). The green curves are the LO result, red NLO. The black lines are NNLO results and are visually indistinguishable from the NLO curves for $Q=30 \mathrm{GeV}$. We set $\mu_{h}=\mu_{f}=Q$, and $b\left(\mu_{f}\right)=4$. For the intermediate scale, we choose $\mu_{i}=M_{X}$ in momentum space and $\mu_{i}=Q / \sqrt{\bar{N}}$ in moment space.

c smaller than the value of $N$ at which the pole occurs. Even with this prescription, the numerical integral is not well behaved in the limit $x \rightarrow 1$, since the damping of the integrand becomes weaker and weaker as $x$ approaches the endpoint. In Figure 9] we compare the results for the $x$-space structure function obtained through numerical Mellin inversion with those obtained directly in momentum space (63). One source of numerical differences arises because the relation (73) is only approximate, ${ }^{4}$ so that the solution to the RG equation for $J_{N}\left(Q^{2}, \mu\right)$ receives corrections which are suppressed as $1 / N$, while our momentum-space solution (46) is exact. Another is that the default choice of the intermediate scale $\mu_{i}$ is different in the two approaches. The numerical differences are noticeable for smaller values of $Q$, but become negligible at $Q=30 \mathrm{GeV}$.

In the effective-theory result for the moments, the Landau pole in the inverse Mellin transformation can be avoided by performing the inversion to $x$-space with the appropriate scale choice for momentum space, $\mu_{i} \approx Q \sqrt{1-x}$, instead of $\mu_{i}=Q / \sqrt{\bar{N}}$. The freedom to choose the scales as appropriate for the quantity under consideration is an important advantage of our approach. The Landau-pole ambiguity in the Mellin inversion is not the only problem that arises from the fact that the scales cannot be varied in the standard resummation formalism. An additional difficulty was pointed out in [5]. To illustrate it, let us consider the structure function at the leading logarithmic level, even though this is not a consistent approximation in RG-improved perturbation theory. Our result (63) then reduces to

$$
K\left(x, Q^{2}, \mu_{f}\right)=\exp \left[4 S\left(\mu_{h}, \mu_{i}\right)+2 a_{\Gamma}\left(\mu_{i}, \mu_{f}\right) \ln (1-x)\right],
$$

\footnotetext{
${ }^{4}$ The exact form of the RG equation obeyed by the jet-function moments can be found in 31.
} 
where we have approximated $\frac{1-x}{x} \approx 1-x$. From (63), we see that we have to choose $\mu_{h} \sim Q$ to make the double logarithms in the perturbative expansion of the hard matching coefficient $C_{V}\left(Q^{2}, \mu\right)$ small. Similarly, to avoid the appearance of large logarithms in the associated jet function $\widetilde{j}\left(\ln \frac{M_{X}^{2}}{\mu_{i}^{2}}, \mu\right)$, the choice $\mu_{i} \sim M_{X}$ is mandatory. Let us now look at the structure function integrated over the endpoint region

$$
\mathcal{F}_{2}^{\mathrm{ns}}\left(x, Q^{2}\right)=\int_{1-x}^{1} d y F_{2}^{\mathrm{ns}}\left(y, Q^{2}\right) .
$$

In this case, the appropriate choice of the intermediate scale for integral $\mathcal{F}_{2}^{\text {ns }}\left(x, Q^{2}\right)$ is $\mu_{i} \sim$ $Q \sqrt{1-x}$, as can be checked by explicitly performing the integral over (63). If one instead chooses the scale $\mu_{i}$ to avoid logarithms on the level of the integrand, then the integral (89) becomes singular. To see the problem, we set $\mu_{f}=\mu_{h}=Q, \mu_{i} \approx Q \sqrt{1-y}$ and, for illustration purposes, approximate the Sudakov factor by expanding it to leading order around fixed coupling $\alpha_{s}(Q)$, as was done in [5]. The integral (89) then becomes

$$
\begin{aligned}
\mathcal{F}_{2}^{\mathrm{ns}}\left(x, Q^{2}\right) & =\int_{1-x}^{1} d y \sum_{q} e_{q}^{2} y \phi_{q}^{\mathrm{ns}}(y, Q) \exp \left[-a \ln ^{2} \frac{\mu_{i}^{2}}{\mu_{h}^{2}}+2 a \ln \frac{\mu_{i}^{2}}{\mu_{f}^{2}} \ln (1-y)\right] \\
& =\int_{1-x}^{1} d y \sum_{q} e_{q}^{2} y \phi_{q}^{\mathrm{ns}}\left(y, \mu_{f}\right) \exp \left[a \ln ^{2}(1-y)\right],
\end{aligned}
$$

with $a=\Gamma_{0} \frac{\alpha_{s}(Q)}{8 \pi}$. Because the exponential factor grows faster than any power as $y \rightarrow 1$, this integral diverges. Its expansion in $a$ is an asymptotic series with factorially growing terms. As was shown in [5] the ambiguity associated with the non-integrable singularity for $y \rightarrow 1$ is of order

$$
e^{-1 / 4 a} \sim\left(\frac{\Lambda_{\mathrm{QCD}}}{Q}\right)^{\frac{\beta_{0}}{4 C_{F}}} \approx\left(\frac{\Lambda_{\mathrm{QCD}}}{Q}\right)^{1.4}
$$

for $n_{f}=5$. In [5] it was shown that the above divergence does not occur if the Sudakov resummation is performed in moment space and the inverse transformation is performed exactly, without dropping subleading logarithms $\ln (1-x)$. From this, the authors concluded that the appropriate place to perform resummations is moment space and that leading logarithmic resummations in $x$-space are problematic. Our analysis shows that it is simply a bad choice of scale that produces the problem of the spurious power correction: the usual moment-space formalism produces logarithms $\ln ^{2} N$ in the Sudakov exponent, which translates into $\ln ^{2}(1-x)$ at leading logarithmic accuracy, which in turn causes the problem in (90). However, the proper way to perform the calculation is to keep the matching scales arbitrary and choose them such that the final result of a given calculation does not contain large logarithms. This avoids the above problem as well as the occurrence of Landau-pole ambiguities in inverse Mellin transforms.

We hope that the above discussion helps to overcome the misconception that Mellin moment space is the "correct place" to perform the threshold resummation, and that resummation in $x$-space leads to inconsistent results. Quite to the contrary, the final analytical formulae we obtain in momentum space are simpler than those derived in moment space, they are free of spurious, unphysical power ambiguities and, as Figure 9 shows, the perturbative expansion in $x$-space exhibits a better apparent convergence. 


\section{$5 \quad$ Summary and conclusions}

We analyzed DIS in the threshold region $x \rightarrow 1$ using SCET. With a detailed analysis in the effective theory, we rederived the standard QCD factorization theorem [1, 2, 3] for the nonsinglet structure function $F_{2}^{\text {ns }}\left(x, Q^{2}\right)$. While this process had been investigated in the effective theory before [17, 18, 19, 20, 21, 22, we argued that previous studies were incomplete. Our analysis resolves the issues left open in these papers. We agree with [18] that in a diagrammatic analysis momentum modes with low virtuality appear. Their presence is a consequence of the fact that in the limit $x \rightarrow 1$ one parton carries nearly all the momentum of the nucleon and the characteristic scale associated with the target remnants is $m^{2}(1-x)$, at least in a perturbative analysis. However, here we have shown that these modes do not translate into non-perturbative $Q$ dependence in the parton distribution function. In [17 it was argued that such momentum regions do not contribute to the effective-theory calculation, and in [20] that they would be screened away non-perturbatively. Since these target remnants are part of the endpoint parton distribution function, it is incorrect to exclude them from the effective-theory factorization analysis, and there is no need to invoke a mechanism which eliminates them. For the same reason, there are no extra "soft" contributions outside the parton distribution function, contrary to what was postulated in [19].

With the factorization theorem at hand, we then performed the resummation of large logarithms by solving the RG equations of the effective theory. Our result involves three scales: a hard matching scale $\mu_{h}$, a jet scale $\mu_{i}$, and the factorization scale $\mu_{f}$. By choosing the perturbative scales to satisfy $\mu_{h} \sim Q$ and $\mu_{i} \sim M_{X}$, we avoid the presence of large logarithms. This approach has several advantages compared to the standard resummation technique for DIS. It enables us to derive a simple analytic expression for the resummed structure function directly in $x$-space, thus circumventing the problems associated with moment-space resummation. We can also estimate the higher-order perturbative uncertainties by varying the matching scales. It is trivial to recover the fixed-order result by setting all scales equal, $\mu_{h}=\mu_{i}=\mu_{f}$. This makes it straightforward to combine our results with fixed-order calculations valid away from the threshold region.

An advantage of the effective-theory approach is that the resummed results are free of Landau-pole ambiguities. In the standard approach, these appear twice: in the resummed exponent in moment space and also in the Mellin inversion back to momentum space. Since we perform the resummation in momentum space by integrating out the higher scales and using RG evolution to go to lower scales, our expressions do not involve the strong coupling constant evaluated at scales below the minimum of the intermediate matching scale and the factorization scale at which the parton distribution function is renormalized. Therefore, Landau-pole ambiguities do not arise at any finite order in perturbation theory. We showed that our results are formally equivalent to the standard ones order by order in perturbation theory. This allowed us to relate the radiation function $B_{q}$ to a combination of the anomalous dimension

$\gamma^{J}$ of the jet function and effective-theory matching coefficients. The two objects $B_{q}$ and $\gamma^{J}$ are identical at leading order, but beyond this the relation is highly non-trivial.

Since the parton distribution functions fall off very rapidly near $x \rightarrow 1$, it is experimentally challenging to measure structure functions at large $x$. For this reason the amount of available experimental information near threshold is very limited. However, because of its relative 
simplicity and since the perturbative quantities are known with high precision, the threshold resummation for DIS has provided us with an ideal setup to develop our formalism. In the future, we plan to use the same approach to perform resummations in other, phenomenologically more relevant situations.

\section{Acknowledgments}

We are grateful to Geoffrey Bodwin, Stefano Catani, and Einan Gardi for useful discussions. The research of T.B. was supported by the Department of Energy under Grant DE-AC0276CH03000. The research of M.N. was supported by the National Science Foundation under Grant PHY-0355005. The work of B.P. was supported by the DFG Sonderforschungsbereich SFB/TR09 "Computational Theoretical Particle Physics". Fermilab is operated by Universities Research Association Inc., under contract with the U.S. Department of Energy. 


\section{Appendix}

The exact solutions (39) to the RG equations (38) can be evaluated by expanding the anomalous dimensions and the QCD $\beta$-function as perturbative series in the strong coupling. We work consistently at NNLO in RG-improved perturbation theory, keeping terms through order $\alpha_{s}^{2}$ in the final expressions for the Sudakov exponent $S$ and the functions $a_{\Gamma}, a_{\gamma^{V}}$, and $a_{\gamma^{J}}$. We define the expansion coefficients as

$$
\begin{aligned}
\Gamma_{\text {cusp }}\left(\alpha_{s}\right) & =\Gamma_{0} \frac{\alpha_{s}}{4 \pi}+\Gamma_{1}\left(\frac{\alpha_{s}}{4 \pi}\right)^{2}+\Gamma_{2}\left(\frac{\alpha_{s}}{4 \pi}\right)^{3}+\Gamma_{3}\left(\frac{\alpha_{s}}{4 \pi}\right)^{4}+\ldots \\
\beta\left(\alpha_{s}\right) & =-2 \alpha_{s}\left[\beta_{0} \frac{\alpha_{s}}{4 \pi}+\beta_{1}\left(\frac{\alpha_{s}}{4 \pi}\right)^{2}+\beta_{2}\left(\frac{\alpha_{s}}{4 \pi}\right)^{3}+\beta_{3}\left(\frac{\alpha_{s}}{4 \pi}\right)^{4}+\ldots\right]
\end{aligned}
$$

and similarly for the other anomalous dimensions. In terms of these quantities, the function $a_{\Gamma}$ is given by

$$
\begin{aligned}
a_{\Gamma}(\nu, \mu)= & \frac{\Gamma_{0}}{2 \beta_{0}}\left\{\ln \frac{\alpha_{s}(\mu)}{\alpha_{s}(\nu)}+\left(\frac{\Gamma_{1}}{\Gamma_{0}}-\frac{\beta_{1}}{\beta_{0}}\right) \frac{\alpha_{s}(\mu)-\alpha_{s}(\nu)}{4 \pi}\right. \\
& \left.+\left[\frac{\Gamma_{2}}{\Gamma_{0}}-\frac{\beta_{2}}{\beta_{0}}-\frac{\beta_{1}}{\beta_{0}}\left(\frac{\Gamma_{1}}{\Gamma_{0}}-\frac{\beta_{1}}{\beta_{0}}\right)\right] \frac{\alpha_{s}^{2}(\mu)-\alpha_{s}^{2}(\nu)}{32 \pi^{2}}+\ldots\right\} .
\end{aligned}
$$

The result for the Sudakov factor $S$ is more complicated. We obtain

$$
\begin{aligned}
S(\nu, \mu)=\frac{\Gamma_{0}}{4 \beta_{0}^{2}}\left\{\frac{4 \pi}{\alpha_{s}(\nu)}\left(1-\frac{1}{r}-\ln r\right)+\left(\frac{\Gamma_{1}}{\Gamma_{0}}-\frac{\beta_{1}}{\beta_{0}}\right)(1-r+\ln r)+\frac{\beta_{1}}{2 \beta_{0}} \ln ^{2} r\right. \\
+\frac{\alpha_{s}(\nu)}{4 \pi}\left[\left(\frac{\beta_{1} \Gamma_{1}}{\beta_{0} \Gamma_{0}}-\frac{\beta_{2}}{\beta_{0}}\right)(1-r+r \ln r)+\left(\frac{\beta_{1}^{2}}{\beta_{0}^{2}}-\frac{\beta_{2}}{\beta_{0}}\right)(1-r) \ln r\right. \\
\left.\quad-\left(\frac{\beta_{1}^{2}}{\beta_{0}^{2}}-\frac{\beta_{2}}{\beta_{0}}-\frac{\beta_{1} \Gamma_{1}}{\beta_{0} \Gamma_{0}}+\frac{\Gamma_{2}}{\Gamma_{0}}\right) \frac{(1-r)^{2}}{2}\right] \\
+\left(\frac{\alpha_{s}(\nu)}{4 \pi}\right)^{2}\left[\left(\frac{\beta_{1} \beta_{2}}{\beta_{0}^{2}}-\frac{\beta_{1}^{3}}{2 \beta_{0}^{3}}-\frac{\beta_{3}}{2 \beta_{0}}+\frac{\beta_{1}}{\beta_{0}}\left(\frac{\Gamma_{2}}{\Gamma_{0}}-\frac{\beta_{2}}{\beta_{0}}+\frac{\beta_{1}^{2}}{\beta_{0}^{2}}-\frac{\beta_{1} \Gamma_{1}}{\beta_{0} \Gamma_{0}}\right) \frac{r^{2}}{2}\right) \ln r\right. \\
+\left(\frac{\Gamma_{3}}{\Gamma_{0}}-\frac{\beta_{3}}{\beta_{0}}+\frac{2 \beta_{1} \beta_{2}}{\beta_{0}^{2}}+\frac{\beta_{1}^{2}}{\beta_{0}^{2}}\left(\frac{\Gamma_{1}}{\Gamma_{0}}-\frac{\beta_{1}}{\beta_{0}}\right)-\frac{\beta_{2} \Gamma_{1}}{\beta_{0} \Gamma_{0}}-\frac{\beta_{1} \Gamma_{2}}{\beta_{0} \Gamma_{0}}\right) \frac{(1-r)^{3}}{3} \\
+\left(\frac{3 \beta_{3}}{4 \beta_{0}}-\frac{\Gamma_{3}}{2 \Gamma_{0}}+\frac{\beta_{1}^{3}}{\beta_{0}^{3}}-\frac{3 \beta_{1}^{2} \Gamma_{1}}{4 \beta_{0}^{2} \Gamma_{0}}+\frac{\beta_{2} \Gamma_{1}}{\beta_{0} \Gamma_{0}}+\frac{\beta_{1} \Gamma_{2}}{4 \beta_{0} \Gamma_{0}}-\frac{7 \beta_{1} \beta_{2}}{4 \beta_{0}^{2}}\right)(1-r)^{2} \\
\left.\left.+\left(\frac{\beta_{1} \beta_{2}}{\beta_{0}^{2}}-\frac{\beta_{3}}{\beta_{0}}-\frac{\beta_{1}^{2} \Gamma_{1}}{\beta_{0}^{2} \Gamma_{0}}+\frac{\beta_{1} \Gamma_{2}}{\beta_{0} \Gamma_{0}}\right) \frac{1-r}{2}\right]+\ldots\right\}
\end{aligned}
$$

where $r=\alpha_{s}(\mu) / \alpha_{s}(\nu)$. Whereas the three-loop anomalous dimensions and $\beta$-function are required in (93), the expression for $S$ also involves the four-loop coefficients $\Gamma_{3}$ and $\beta_{3}$. 
We now list expressions for the anomalous dimensions and the QCD $\beta$-function, quoting all results in the $\overline{\mathrm{MS}}$ renormalization scheme. For the convenience of the reader, we also give numerical results for $n_{f}=5$. The expansion of the cusp anomalous dimension $\Gamma_{\text {cusp }}$ to twoloop order was obtained some time ago [10, while recently the three-loop coefficient has been obtained in [46]. For the four-loop coefficient $\Gamma_{3}$, we use the Padé approximants derived in 44. The results are

$$
\begin{aligned}
\Gamma_{0}= & 4 C_{F}=\frac{16}{3}, \\
\Gamma_{1}= & 4 C_{F}\left[\left(\frac{67}{9}-\frac{\pi^{2}}{3}\right) C_{A}-\frac{20}{9} T_{F} n_{f}\right] \approx 36.8436, \\
\Gamma_{2}= & 4 C_{F}\left[C_{A}^{2}\left(\frac{245}{6}-\frac{134 \pi^{2}}{27}+\frac{11 \pi^{4}}{45}+\frac{22}{3} \zeta_{3}\right)+C_{A} T_{F} n_{f}\left(-\frac{418}{27}+\frac{40 \pi^{2}}{27}-\frac{56}{3} \zeta_{3}\right)\right. \\
& \left.+C_{F} T_{F} n_{f}\left(-\frac{55}{3}+16 \zeta_{3}\right)-\frac{16}{27} T_{F}^{2} n_{f}^{2}\right] \approx 239.208, \\
\Gamma_{3} \approx & 7849,4313,1553 \text { for } n_{f}=3,4,5 .
\end{aligned}
$$

The anomalous dimension $\gamma^{V}$ can be determined up to three-loop order from the partial threeloop expression for the on-shell quark form factor in $\mathrm{QCD}$, which has recently been obtained in [45]. We find

$$
\begin{aligned}
\gamma_{0}^{V}= & -6 C_{F}=-8 \\
\gamma_{1}^{V}= & C_{F}^{2}\left(-3+4 \pi^{2}-48 \zeta_{3}\right)+C_{F} C_{A}\left(-\frac{961}{27}-\frac{11 \pi^{2}}{3}+52 \zeta_{3}\right)+C_{F} T_{F} n_{f}\left(\frac{260}{27}+\frac{4 \pi^{2}}{3}\right) \\
\approx & 1.1419 \\
\gamma_{2}^{V}= & C_{F}^{3}\left(-29-6 \pi^{2}-\frac{16 \pi^{4}}{5}-136 \zeta_{3}+\frac{32 \pi^{2}}{3} \zeta_{3}+480 \zeta_{5}\right) \\
& +C_{F}^{2} C_{A}\left(-\frac{151}{2}+\frac{410 \pi^{2}}{9}+\frac{494 \pi^{4}}{135}-\frac{1688}{3} \zeta_{3}-\frac{16 \pi^{2}}{3} \zeta_{3}-240 \zeta_{5}\right) \\
& +C_{F} C_{A}^{2}\left(-\frac{139345}{1458}-\frac{7163 \pi^{2}}{243}-\frac{83 \pi^{4}}{45}+\frac{7052}{9} \zeta_{3}-\frac{88 \pi^{2}}{9} \zeta_{3}-272 \zeta_{5}\right) \\
& +C_{F}^{2} T_{F} n_{f}\left(\frac{5906}{27}-\frac{52 \pi^{2}}{9}-\frac{56 \pi^{4}}{27}+\frac{1024}{9} \zeta_{3}\right) \\
& +C_{F} C_{A} T_{F} n_{f}\left(-\frac{34636}{729}+\frac{5188 \pi^{2}}{243}+\frac{44 \pi^{4}}{45}-\frac{3856}{27} \zeta_{3}\right) \\
& +C_{F} T_{F}^{2} n_{f}^{2}\left(\frac{19336}{729}-\frac{80 \pi^{2}}{27}-\frac{64}{27} \zeta_{3}\right) \approx-249.388 .
\end{aligned}
$$


The results for the expansion coefficients of the jet-function anomalous dimension $\gamma^{J}$ are

$$
\begin{aligned}
\gamma_{0}^{J}= & -3 C_{F}=-4 \\
\gamma_{1}^{J}= & C_{F}^{2}\left(-\frac{3}{2}+2 \pi^{2}-24 \zeta_{3}\right)+C_{F} C_{A}\left(-\frac{1769}{54}-\frac{11 \pi^{2}}{9}+40 \zeta_{3}\right)+C_{F} T_{F} n_{f}\left(\frac{242}{27}+\frac{4 \pi^{2}}{9}\right) \\
\approx & 38.6763 \\
\gamma_{2}^{J}= & C_{F}^{3}\left(-\frac{29}{2}-3 \pi^{2}-\frac{8 \pi^{4}}{5}-68 \zeta_{3}+\frac{16 \pi^{2}}{3} \zeta_{3}+240 \zeta_{5}\right) \\
& +C_{F}^{2} C_{A}\left(-\frac{151}{4}+\frac{205 \pi^{2}}{9}+\frac{247 \pi^{4}}{135}-\frac{844}{3} \zeta_{3}-\frac{8 \pi^{2}}{3} \zeta_{3}-120 \zeta_{5}\right) \\
& +C_{F} C_{A}^{2}\left(-\frac{412907}{2916}-\frac{419 \pi^{2}}{243}-\frac{19 \pi^{4}}{10}+\frac{5500}{9} \zeta_{3}-\frac{88 \pi^{2}}{9} \zeta_{3}-232 \zeta_{5}\right) \\
& +C_{F}^{2} T_{F} n_{f}\left(\frac{4664}{27}-\frac{32 \pi^{2}}{9}-\frac{164 \pi^{4}}{135}+\frac{208}{9} \zeta_{3}\right) \\
& +C_{F} C_{A} T_{F} n_{f}\left(-\frac{5476}{729}+\frac{1180 \pi^{2}}{243}+\frac{46 \pi^{4}}{45}-\frac{2656}{27} \zeta_{3}\right) \\
& +C_{F} T_{F}^{2} n_{f}^{2}\left(\frac{13828}{729}-\frac{80 \pi^{2}}{81}-\frac{256}{27} \zeta_{3}\right) 204.816 .
\end{aligned}
$$

Finally, the expansion coefficients for the QCD $\beta$-function to four-loop order are

$$
\begin{aligned}
\beta_{0} & =\frac{11}{3} C_{A}-\frac{4}{3} T_{F} n_{f}=\frac{23}{3} \\
\beta_{1} & =\frac{34}{3} C_{A}^{2}-\frac{20}{3} C_{A} T_{F} n_{f}-4 C_{F} T_{F} n_{f} \approx 38.6667 \\
\beta_{2} & =\frac{2857}{54} C_{A}^{3}+\left(2 C_{F}^{2}-\frac{205}{9} C_{F} C_{A}-\frac{1415}{27} C_{A}^{2}\right) T_{F} n_{f}+\left(\frac{44}{9} C_{F}+\frac{158}{27} C_{A}\right) T_{F}^{2} n_{f}^{2} \\
& \approx 180.907, \\
\beta_{3} & =\frac{149753}{6}+3564 \zeta_{3}-\left(\frac{1078361}{162}+\frac{6508}{27} \zeta_{3}\right) n_{f}+\left(\frac{50065}{162}+\frac{6472}{81} \zeta_{3}\right) n_{f}^{2}+\frac{1093}{729} n_{f}^{3} \\
& \approx 4826.16,
\end{aligned}
$$

where the value of $\beta_{3}$ is taken from [54] and corresponds to $N_{c}=3$ and $T_{F}=\frac{1}{2}$. 


\section{References}

[1] G. Sterman, Nucl. Phys. B 281, 310 (1987).

[2] S. Catani and L. Trentadue, Nucl. Phys. B 327, 323 (1989).

[3] G. P. Korchemsky and G. Marchesini, Nucl. Phys. B 406, 225 (1993) hep-ph/9210281.

[4] S. Moch, J. A. M. Vermaseren and A. Vogt, Nucl. Phys. B 726, 317 (2005) hep-ph/0506288.

[5] S. Catani, M. L. Mangano, P. Nason and L. Trentadue, Nucl. Phys. B 478, 273 (1996) hep-ph/9604351.

[6] A. Vogt, Phys. Lett. B 471, 97 (1999) hep-ph/9910545.

[7] M. Beneke and V. M. Braun, Nucl. Phys. B 454, 253 (1995) hep-ph/9506452.

[8] G. P. Korchemsky and G. Marchesini, Phys. Lett. B 313, 433 (1993).

[9] G. P. Korchemsky and A. V. Radyushkin, Nucl. Phys. B 283, 342 (1987).

[10] I. A. Korchemskaya and G. P. Korchemsky, Phys. Lett. B 287, 169 (1992).

[11] C. W. Bauer, S. Fleming, D. Pirjol and I. W. Stewart, Phys. Rev. D 63, 114020 (2001) hep-ph/0011336.

[12] C. W. Bauer, D. Pirjol and I. W. Stewart, Phys. Rev. D 65, 054022 (2002) hep-ph/0109045.

[13] M. Beneke, A. P. Chapovsky, M. Diehl and T. Feldmann, Nucl. Phys. B 643, 431 (2002) hep-ph/0206152.

[14] R. J. Hill and M. Neubert, Nucl. Phys. B 657, 229 (2003) hep-ph/0211018.

[15] T. Becher and M. Neubert, hep-ph/0605050.

[16] C. W. Bauer, S. Fleming, D. Pirjol, I. Z. Rothstein and I. W. Stewart, Phys. Rev. D 66, 014017 (2002) hep-ph/0202088.

[17] A. V. Manohar, Phys. Rev. D 68, 114019 (2003) hep-ph/0309176.

[18] B. D. Pecjak, JHEP 0510, 040 (2005) hep-ph/0506269.

[19] J. Chay and C. Kim, hep-ph/0511066.

[20] A. V. Manohar, Phys. Lett. B 633, 729 (2006) hep-ph/0512173.

[21] A. Idilbi, X. d. Ji and F. Yuan, hep-ph/0605068.

[22] P. Chen, A. Idilbi and X. Ji, hep-ph/0607003. 
[23] A. Idilbi and X. d. Ji, Phys. Rev. D 72, 054016 (2005) hep-ph/0501006.

[24] M. Neubert, Eur. Phys. J. C 40, 165 (2005) hep-ph/0408179.

[25] M. Neubert, Phys. Rev. D 72, 074025 (2005) hep-ph/0506245.

[26] S. W. Bosch, R. J. Hill, B. O. Lange and M. Neubert, Phys. Rev. D 67, 094014 (2003) hep-ph/0301123.

[27] T. Becher, R. J. Hill and M. Neubert, Phys. Rev. D 69, 054017 (2004) hep-ph/0308122.

[28] C. W. Bauer and A. V. Manohar, Phys. Rev. D 70, 034024 (2004) hep-ph/0312109.

[29] T. Becher, R. J. Hill, B. O. Lange and M. Neubert, Phys. Rev. D 69, 034013 (2004) hep-ph/0309227.

[30] M. Beneke and T. Feldmann, Phys. Lett. B 553, 267 (2003) hep-ph/0211358.

[31] T. Becher and M. Neubert, Phys. Lett. B 637, 251 (2006) hep-ph/0603140.

[32] S. W. Bosch, B. O. Lange, M. Neubert and G. Paz, Nucl. Phys. B 699, 335 (2004) hep-ph/0402094.

[33] J. C. Collins and D. E. Soper, Nucl. Phys. B 194, 445 (1982).

[34] K. S. M. Lee and I. W. Stewart, Nucl. Phys. B 721, 325 (2005) hep-ph/0409045.

[35] S. W. Bosch, M. Neubert and G. Paz, JHEP 0411, 073 (2004) hep-ph/0409115.

[36] M. Beneke, F. Campanario, T. Mannel and B. D. Pecjak, JHEP 0506, 071 (2005) hep-ph/0411395.

[37] R. Akhoury, M. G. Sotiropoulos and G. Sterman, Phys. Rev. Lett. 81, 3819 (1998) hep-ph/9807330.

[38] B. O. Lange and M. Neubert, Phys. Rev. Lett. 91, 102001 (2003) hep-ph/0303082.

[39] F. De Fazio and M. Neubert, JHEP 9906, 017 (1999) hep-ph/9905351.

[40] S. J. Lee and M. Neubert, Phys. Rev. D 72, 094028 (2005) hep-ph/0509350.

[41] G. Kramer and B. Lampe, Z. Phys. C 34, 497 (1987) [Erratum-ibid. C 42, 504 (1989)].

[42] T. Matsuura and W. L. van Neerven, Z. Phys. C 38, 623 (1988).

[43] T. Matsuura, S. C. van der Marck and W. L. van Neerven, Nucl. Phys. B 319, 570 (1989).

[44] T. Gehrmann, T. Huber and D. Maitre, Phys. Lett. B 622, 295 (2005) hep-ph/0507061.

[45] S. Moch, J. A. M. Vermaseren and A. Vogt, JHEP 0508, 049 (2005) hep-ph/0507039. 
[46] S. Moch, J. A. M. Vermaseren and A. Vogt, Nucl. Phys. B 688, 101 (2004) hep-ph/0403192.

[47] See e.g. the HEPDATA database: http://durpdg.dur.ac.uk/HEPDATA

[48] R. G. Roberts and M. R. Whalley, J. Phys. G 17, D1 (1991).

[49] T. Gehrmann, R. G. Roberts and M. R. Whalley, J. Phys. G 25, A1 (1999).

[50] L. W. Whitlow, E. M. Riordan, S. Dasu, S. Rock and A. Bodek, Phys. Lett. B 282, 475 (1992).

[51] A. C. Benvenuti et al. [BCDMS Collaboration], Phys. Lett. B 237, 599 (1990).

[52] J. F. Gunion, Phys. Rev. D 10, 242 (1974).

[53] R. Blankenbecler and S. J. Brodsky, Phys. Rev. D 10, 2973 (1974).

[54] T. van Ritbergen, J. A. M. Vermaseren and S. A. Larin, Phys. Lett. B 400, 379 (1997) hep-ph/9701390. 